\title{
Rigid nongeometric orientifolds and the swampland
}

\author{
Pramod Shukla๑* \\ ICTP, Strada Costiera 11, Trieste 34151, Italy
}

(Received 18 May 2020; accepted 22 February 2021; published 15 April 2021)

\begin{abstract}
Nongeometric flux compactifications with frozen complex structure moduli have been recently studied for several phenomenological purposes. In this context, we analyze the possibility of realizing de Sitter solutions in the context of $\mathcal{N}=1$ type II nongeometric flux compactifications using the $\mathbb{T}^{6} /\left(\mathbb{Z}_{3} \times \mathbb{Z}_{3}\right)$ toroidal orientifolds. For the type IIB case, we observe that the Bianchi identities are too strong to simultaneously allow both the NS-NS three-form flux $\left(H_{3}\right)$ and the nongeometric $(Q)$ flux to take nonzero values, which makes this model irrelevant for phenomenology due to the no-scale structure. For the type IIA case, we find that all the (nongeometric) flux solutions satisfying the Bianchi identities result in de Sitter no-go scenarios except for one case in which the no-go condition can be evaded. However for this case also, in our (limited) numerical investigation we do not find any de Sitter vacua using the integer fluxes satisfying all the Bianchi identities.
\end{abstract}

DOI: 10.1103/PhysRevD.103.086010

\section{INTRODUCTION}

In the context of moduli stabilization, the fourdimensional effective potentials arising from type II flux compactifications have attracted a lot attention since more than a decade [1-11]. In particular, the study of nongeometric flux compactifications and their four-dimensional scalar potentials have led to a continuous progress in various phenomenological aspects such as towards moduli stabilization, in constructing de Sitter vacua and also in realizing the minimal aspects of inflationary cosmology [12-30]. In the context of type II supergravity theories, such (non)geometric fluxes arise from a successive application of the $T$ duality on the NS-NS three-form flux $\mathrm{H}_{3}$ [31-37], and the exciting part about studying these fluxes is the fact that they can generically induce tree level contributions to the scalar potential for all the moduli and hence can subsequently help in dynamically stabilizing them through the lowest order effects. Moreover, the common presence of the nongeometric fluxes in double field theory (DFT), superstring flux compactifications, and the gauged supergravities has helped in understanding a variety of interconnecting aspects in these three formulations along with opening new windows for exploring some phenomenological aspects as well [12,16,34-53]. Moreover, the nongeometric flux compactification scenarios also present

\footnotetext{
*pramodmaths@gmail.com
}

Published by the American Physical Society under the terms of the Creative Commons Attribution 4.0 International license. Further distribution of this work must maintain attribution to the author(s) and the published article's title, journal citation, and DOI. Funded by SCOAP. some interesting utilizations of the symplectic geometries $[54,55]$ to formulate the effective scalar potentials; e.g., see [56-58], which generalize the work of [2,3] by including the nongeometric fluxes. The ten-dimensional origin of the four-dimensional nongeometric scalar potentials have been explored recently via an iterative series of works in the supergravity theories [44-47,49,50,55,59-63] and through some robust realization of the DFT reduction on Calabi Yau threefolds [64]. Moreover, a concrete connection among the type II effective potentials derived from DFT reductions and those of the symplectic approach has been established in $[56,57]$. A recent review on the developments in the nongeometric flux compactifications can be found in [65].

Although a consistent incorporation of the various nongeometric fluxes enriches the compactification backgrounds creating more/better possibilities for model building, it is still not fully known how many and which type of fluxes can be simultaneously turned on. However, it has been observed through explicit constructions that it is quite constraining to consistently satisfy all the quadratic flux constraints coming from the various Bianchi identities and the tadpole cancellation conditions. In this regard, there have been two formulations for computing the Bianchi identities; the standard one mostly applicable to toroidal orientifolds involves fluxes with noncohomology indices $[14,17,36]$, while in the cohomology formulation fluxes are represented using the nontrivial cohomology indices $[14,66,67]$. For obvious reasons the later one is utilized for simplifying the scalar potentials in the models developed in the beyond toroidal settings. However, a mismatch between the two sets of constraints arising from the Bianchi identities of these two formulations have been observed in $[14,51]$ and studied in some good detail in $[68,69]$. 
The additional identities in the cohomology formulation might be relevant in the recent interesting studies performed in [25-29,70].

In the context of type IIA flux compactifications, some significant initiatives for the moduli stabilization and the study of scalar potential have been taken, e.g., see $[4,13,14,50,71-77]$, and, in the meantime, several no-go scenarios forbidding de Sitter and inflationary realizations have been also found [78-97]. In close connections with these type IIA no-go scenarios, the swampland conjecture of [98] about obstructing the de Sitter realization in a consistent theory of quantum gravity has been recently promoted as a bound involving the scalar potential $(V)$ and its derivatives [99],

$$
|\nabla V| \geq \frac{c}{M_{p}} V
$$

where the constant $c>0$ is an order one quantity. This bound in Eq. (1.1) does not only forbid the de Sitter minima but also the de Sitter maxima as well, and several counter examples were known [81,87,100-102] or have been proposed soon after the proposal was made [103-113] reflecting the need of refining the conjecture. Subsequently a refined version of the conjecture has been proposed which states that at least one of the following two constraints should always hold [114],

$$
|\nabla V| \geq \frac{c}{M_{p}} V, \quad \min \left[\nabla_{i} \nabla_{j} V\right] \leq-\frac{c^{\prime}}{M_{p}^{2}} V,
$$

where $c$ and $c^{\prime}>0$ are order one constants. Following from the lhs of Eq. (1.2), one can observe that the conjecture is trivial for nonpositive potentials, as well as in the limit $M_{p} \rightarrow \infty$ when gravity decouples. Moreover, these two parameters $c$ and $c^{\prime}$ are correlated with the well-known inflationary parameters expressed in terms of the derivatives of the potential, namely the $\epsilon_{V}$ and $\eta_{V}$ parameters; e.g., see [80,115], where $\epsilon_{V} \geq \frac{1}{2} c^{2}$ and $\left|\eta_{V}\right| \geq c^{\prime}$, and these parameters are needed to be sufficiently smaller than one, i.e., $\epsilon_{V} \ll 1$ and $\left|\eta_{V}\right| \ll 1$, for having the slow-roll inflation. In the meanwhile, there have been a surge in related studies in connection with this conjecture [103-113, 116-127]; see [128] also for a recent review. The swampland conjecture [98] has been also found to be in close connections with the allowed inflaton field range in a trustworthy effective field theory description as it has been argued that massive tower of states can get excited after a certain limit to the inflaton excursions [129-143]. However, let us also note that in contrary to the (minimal) de Sitter no-go scenarios, there have been tremendous amount of efforts leading to several proposals for realizing stable de Sitter vacua in the context of string model building $[1,144-155]$; see $[156,157]$ also for the
$F$-theoretic initiatives taken in this regard. We also refer the interested readers to a recent review in [158].

Toroidal orientifolds have been utilized as basic toolkits for several model building purposes. Being simple they facilitate a playground for performing explicit computations for nongeometric flux compactifications as well. In connection to that, nongeometric flux compactifications with rigid [Calabi Yau (CY)] threefolds have recently witnessed a significant amount of interest in studying some phenomenological aspects $[25,26,28,60,159]$. In the absence of any active complex structure moduli, these setups are quite simple for explicit computations. In this article we consider one such rigid construction using the $\mathbb{T}^{6} /\left(\mathbb{Z}_{3} \times \mathbb{Z}_{3}\right)$ orbifold with the aim to perform a deep investigation on finding de Sitter vacua using nongeometric fluxes satisfying all the NSNS Bianchi identities. For that purpose, first we will explore the mismatch of identities for the $\mathcal{N}=2$ rigid compactifications using the $\mathbb{T}^{6} /\left(\mathbb{Z}_{3} \times \mathbb{Z}_{3}\right)$ orbifold, and subsequently for the $\mathcal{N}=1$ type IIA and type IIB orientifolds to be used later on. It has been a quite conventional anticipation that the inclusion of nongeometric fluxes can (always) provide a window for evading the classical de Sitter no-go theorems; however, using the $T$-dual dictionary of [160] and the mirror arguments, several no-go scenarios have been derived in the context of nongeometric type IIB compactifications in [161]. However, given the fact that mirror of a rigid CY manifold is not a CY space [162-164], a separate analysis for the type II scalar potentials obtained from the rigid compactifications is necessary to explore along the lines of de Sitter swampland conjecture, and we aim to fill this gap in this article.

The article is organized as follows: in Sec. II we present a short review on the generic type II nongeometric flux compactifications with the necessary ingredients relevant for the upcoming sections. Section III provides an explicit computation of the $\mathcal{N}=2$ Bianchi identities appearing in the type II orbifold compactification using a $\mathbb{t}^{6} /\left(\mathbb{Z}_{3} \times \mathbb{Z}_{3}\right)$ threefold, where we also present the full set of flux constraints for the orientifolded type IIA and type IIB theories. Section IV contains some detailed investigation of the de Sitter no-go scenarios in type IIA nongeometric compactifications using the above toroidal model. Section V presents summary and conclusions. In addition, we have Appendices A and B at the end of the article, in which Appendix A includes some details on the explicit computations for the allowed flux components, identities etc. for the $\mathbb{T}^{6} /\left(\mathbb{Z}_{3} \times \mathbb{Z}_{3}\right)$ orientifolds while Appendix B presents a concise review on the type IIA scalar potential that we use in Sec. IV for studying the no-gos in rigid orientifolds in particular.

\section{NONGEOMETRIC FLUX COMPACTIFICATIONS}

In the generic $\mathcal{N}=2$ type II compactifications to four dimensions, let us first fix the notations and conventions. 
We consider $\mu_{A}$ as the basis of harmonic $(1,1)$ forms and $\tilde{\mu}^{A}$ as the respective basis of the dual $(2,2)$ forms. Further, $\mathcal{A}_{\Lambda}$ and $\mathcal{B}^{\Delta}$ form the bases of $(2,1)$ forms. In addition, we denote the zero form as $\mathbf{1}$ and the six form as $\Phi_{6}$. Further, the triple intersection numbers and the normalization of the various forms are fixed as follows

$$
\begin{aligned}
\int_{X_{3}} \mu_{A} \wedge \tilde{\mu}^{B} & \equiv \delta_{A}{ }^{B}, & & \int_{X_{3}} \mathcal{A}_{\Lambda} \wedge \mathcal{B}^{\Delta}=\delta_{\Lambda}{ }^{\Delta}, \\
\int_{X_{3}} \mu_{A} \wedge \mu_{B} \wedge \mu_{C} & =\kappa_{A B C}, & & \int_{X_{3}} \Phi_{6}=1 .
\end{aligned}
$$

To incorporate the effects of various NS-NS fluxes, we consider the twisted differential operator $\mathcal{D}$ defined as follows,

$$
\mathcal{D}=d-H \wedge .-\omega \triangleleft .-Q \triangleright .-R \cdot .,
$$

where the origin of geometric flux $(\omega)$ and the nongeometric fluxes $(Q$ and $R$ ) are motivated by a successive application of $T$ duality on the three-form $H$ flux of the type II supergravities. It turns out that a chain with geometric and nongeometric fluxes appears in the following manner under three $T$-duality transformations [36],

$$
H_{i j k} \rightarrow \omega_{i j}^{k} \rightarrow Q_{i}^{j k} \rightarrow R^{i j k}
$$

Note that the action of various (non)geometric fluxes in Eq. (2.2) are introduced via operations $\triangleleft, \triangleright$, and $\bullet$ on a $p$ form, which changes it into a $(p+1)$ form, a $(p-1)$ form, and a $(p-3)$ form, respectively. The explicit forms of the various flux actions on a $p$ form $A_{p}$ can be given as follows [51,165]:

$$
\begin{aligned}
(\omega \triangleleft A)_{i_{1} i_{2} \ldots i_{p+1}} & =\frac{p(p+1)}{2} \omega_{\left[\underline{i_{1} i_{2}}\right.}{ }^{j} A_{\left.j \mid i_{3} \ldots \ldots i_{p+1}\right]}, \\
(Q \triangleright A)_{i_{1} i_{2} \ldots i_{p-1}} & =\frac{p-1}{2} Q_{\left[\underline{i_{1}}\right.}{ }^{j k} A_{\left.j k \mid i_{2} \ldots \ldots i_{p-1}\right]}, \\
(R \bullet A)_{i_{1} i_{2} \ldots i_{p-3}} & =\frac{p-3}{3 !} R^{j k l} A_{j k l i_{1} \ldots \ldots i_{p-3}},
\end{aligned}
$$

where the underlined indices inside the brackets [..] are antisymmetrized, and in addition we have assumed that the components of $\omega$ flux and the $Q$ flux with one free index are absent, i.e., $\omega_{i j}{ }^{i}=0$ and $Q_{i}{ }^{i j}=0$. This is something called as "tracelessness condition" [37,165] and in the literature it is very common to impose this constraint, especially for the case of a CY compactification as a CY manifold does not have any homologically nontrivial one as well as five cycles, and hence, for the CY orientifold cases, it would be well justified to require that all flux components having effectively one (real six-dimensional) free index to be trivial.

\section{A. Cohomology formulation}

In order to study the nongeometric flux models beyond the toroidal settings, it is important to express the ingredients in what we call as "cohomology formulation." In this formulation, the nontrivial flux actions on various harmonic forms can be given as [67]

$$
\begin{aligned}
& H \wedge \mathbf{1}=-H^{\Lambda} \mathcal{A}_{\Lambda}+H_{\Lambda} \mathcal{B}^{\Lambda}, \quad H \wedge \mathcal{A}_{\Lambda}=-H_{\Lambda} \Phi_{6}, \quad H \wedge \mathcal{B}^{\Lambda}=-H^{\Lambda} \Phi_{6}, \\
& \omega \triangleleft \mu_{A}=\omega_{A}{ }^{\Lambda} \mathcal{A}_{\Lambda}-\omega_{A \Lambda} \mathcal{B}^{\Lambda}, \quad \omega \triangleleft \mathcal{A}_{\Lambda}=-\omega_{A \Lambda} \tilde{\mu}^{A}, \quad \omega \triangleleft \mathcal{B}^{\Lambda}=-\omega_{A}{ }^{\Lambda} \tilde{\mu}^{A}, \\
& Q \triangleright \tilde{\mu}^{A}=-Q^{A \Lambda} \mathcal{A}_{\Lambda}+Q^{A}{ }_{\Lambda} \mathcal{B}^{\Lambda}, \quad Q \triangleright \mathcal{A}_{\Lambda}=-Q^{A}{ }_{\Lambda} \mu_{A}, \quad Q \triangleright \mathcal{B}^{\Lambda}=-Q^{A \Lambda} \mu_{A}, \\
& R \cdot \Phi_{6}=R^{\Lambda} \mathcal{A}_{\Lambda}-R_{\Lambda} \mathcal{B}^{\Lambda}, \quad R \cdot \mathcal{A}_{\Lambda}=-R_{\Lambda} \mathbf{1}, \quad R \cdot \mathcal{B}^{\Lambda}=-R^{\Lambda} \mathbf{1} .
\end{aligned}
$$

Some rough estimates on the counting of the (maximum) possible twist-invariant flux components in cohomology formulation are presented in Table I. Moreover, after the orientifolding is imposed, it is easy to convince that the number of flux components in the cohomology formulation can be simply given as $2\left(h^{1,1}+1\right)\left(h^{2,1}+1\right)$, which is half of the number before orientifolding [14,69]. However, all these estimates and the countings would be further constrained by the Bianchi identities and the tadpole cancellation conditions as we will discuss in the upcoming sections.

In addition, let us also mention the following moduli space metrics relevant for writing down the effective scalar potentials in the four-dimensional theory [5],
TABLE I. Maximum possible number of flux components in cohomology formulation.

\begin{tabular}{lcc}
\hline \hline $\begin{array}{l}\text { Flux type } \\
\text { (standard) }\end{array}$ & $\begin{array}{c}\text { Flux type } \\
\text { (cohomology) }\end{array}$ & $\begin{array}{c}\text { Max. number of } \\
\text { flux components }\end{array}$ \\
\hline$H_{i j k}$ & $H_{\Lambda}, H^{\Lambda}$ & $2\left(h^{2,1}+1\right)$ \\
$\omega_{i j}{ }^{k}$ & $\omega_{A \Lambda}, \omega_{A}{ }^{\Lambda}$ & $2 h^{1,1}\left(h^{2,1}+1\right)$ \\
$Q_{i}{ }^{j k}$ & $Q^{A}{ }_{\Lambda}, Q^{A \Lambda}$ & $2 h^{1,1}\left(h^{2,1}+1\right)$ \\
$R^{i j k}$ & $R_{\Lambda}, R^{\Lambda}$ & $2\left(h^{2,1}+1\right)$ \\
& Total & $4\left(h^{1,1}+1\right)\left(h^{2,1}+1\right)$ \\
\hline \hline
\end{tabular}




$$
\begin{aligned}
& \int_{X_{3}} \mu_{A} \wedge * \mu_{B}=\mathcal{G}_{A B}, \\
& \int_{X_{3}} \tilde{\mu}^{A} \wedge * \tilde{\mu}^{B}=\mathcal{G}^{A B}, \\
& \int_{X_{3}} \beta^{I} \wedge * \beta^{J}=\operatorname{Im} \mathcal{M}^{I J}, \\
& \int_{X_{3}} \alpha_{I} \wedge * \beta^{J}=\operatorname{Re} \mathcal{M}_{I K} \operatorname{Im} \mathcal{M}^{K J}, \\
& \int_{X_{3}} \alpha_{I} \wedge * \alpha_{J}=\left(\operatorname{Im} \mathcal{M}_{I J}+\operatorname{Re} \mathcal{M}_{I K} \operatorname{Im} \mathcal{M}^{K L} \operatorname{Re} \mathcal{M}_{L J}\right),
\end{aligned}
$$

where

$$
\begin{aligned}
K_{A \bar{B}} & =\frac{\kappa_{A} \kappa_{B}-4 \mathcal{V} \kappa_{A B}}{16 \mathcal{V}^{2}} \equiv \frac{1}{4 \mathcal{V}} \mathcal{G}_{A B} \\
K^{A \bar{B}} & =2 t^{A} t^{B}-4 \mathcal{V} \kappa^{A B} \equiv 4 \mathcal{V} \mathcal{G}^{A B} \\
\mathcal{M}_{I J} & =\overline{\mathcal{F}}_{I J}+2 i \frac{(\operatorname{Im} \mathcal{F})_{I K} \mathcal{X}^{K}(\operatorname{Im} \mathcal{F})_{J L} \mathcal{X}^{L}}{\mathcal{X}^{K}(\operatorname{Im} \mathcal{F})_{K L} \mathcal{X}^{L}}
\end{aligned}
$$

Here $K$ is the Kähler potential depending the Kähler moduli $T^{A}=t^{A}+i b^{A}$ where $t^{A}$ denotes the volume of the twocycle moduli and $b^{A}$ denotes the NS-NS $B_{2}$ axions. Further, $\mathcal{M}_{I J}$ presents the moduli space metric depending on the complex structure moduli written in terms of the derivatives of a prepotential $\mathcal{F}$, which is a homogeneous function of degree two in the complex coordinates $\mathcal{X}^{I}$. In addition, the shorthand notations such as $\kappa_{A} t^{A}=6 \mathcal{V}=\kappa_{A B C} t^{A} t^{B} t^{C}$, $\kappa_{A B}=\kappa_{A B C} t^{C}, \kappa_{A}=\kappa_{A B C} t^{B} t^{C}=2 \sigma_{A}$, as well as $\kappa^{A B}$ as the inverse of $\kappa_{A B}$, will be used whenever needed.

\section{B. Bianchi identities}

For studying moduli stabilization and any subsequent phenomenology, a very crucial step to follow is to impose the constraints from various NS-NS Bianchi identities as well as RR tadpoles to get the true nonvanishing contribution to the effective four-dimensional scalar potential. We have two formulations for representing the (NS-NS) Bianchi identities, and we emphasize here that both sets of Bianchi identities have their own advantages and limitations. In the "cohomology formulation" fluxes are expressed in terms of cohomology indices counted via respective Hodge numbers while in the "standard formulation," all the fluxes are written out using the real sixdimensional indices, e.g., $H_{i j k}$ etc.

Note that the various flux actions on the harmonic forms given in Eq. (2.5) can be rewritten in a more compact manner by introducing a couple of multiforms and accordingly clubbing some flux components given as $[64,67]$

$$
\begin{aligned}
\mu_{\mathbf{A}} & =\left\{\Phi_{6}, \mu_{A}\right\}, \quad \tilde{\mu}^{\mathbf{A}}=\left\{\mathbf{1}, \tilde{\mu}^{A}\right\}, \\
\omega_{0 \Lambda} & =R_{\Lambda}, \quad \omega_{0}{ }^{\Lambda}=R^{\Lambda}, \quad Q^{0}{ }_{\Lambda}=H_{\Lambda}, \quad Q^{0 \Lambda}=H^{\Lambda} .
\end{aligned}
$$

Subsequently, the flux actions in Eq. (2.5) can be expressed using the twisted differential $\mathcal{D}$ in the following simpler form,

$$
\begin{aligned}
\mathcal{D} \mathcal{A}_{\Lambda} & =Q^{\mathbf{A}}{ }_{\Lambda} \mu_{\mathbf{A}}+\omega_{\mathbf{A} \Lambda} \tilde{\mu}^{\mathbf{A}}, \quad \mathcal{D} \mathcal{B}^{\Lambda}=Q^{\mathbf{A} \Lambda} \mu_{\mathbf{A}}+\omega_{\mathbf{A}}{ }^{\Lambda} \tilde{\mu}^{\mathbf{A}}, \\
\mathcal{D} \mu_{\mathbf{A}} & =-\omega_{\mathbf{A}}{ }^{\Lambda} \mathcal{A}_{\Lambda}+\omega_{\mathbf{A} \Lambda} \mathcal{B}^{\Lambda}, \quad \mathcal{D} \tilde{\mu}^{\mathbf{A}}=Q^{\mathbf{A} \Lambda} \mathcal{A}_{\Lambda}+Q^{\mathbf{A}}{ }_{\Lambda} \mathcal{B}^{\Lambda} .
\end{aligned}
$$

Imposing the nilpotency of twisted differential operator $\mathcal{D}$ via $\mathcal{D}^{2} A_{p}=0$ where $A_{p}$ corresponds to the various harmonic forms, and using the flux actions in Eq. (2.9) one finds the following six quadratic flux constraints [67],

$$
\begin{array}{lll}
\omega_{\mathbf{A} \Lambda} \omega_{\mathbf{B}}{ }^{\Lambda}=\omega_{\mathbf{B} \Lambda} \omega_{\mathbf{A}}{ }^{\Lambda}, & Q^{\mathbf{A}}{ }_{\Lambda} Q^{\mathbf{B} \Lambda}=Q^{\mathbf{B}}{ }_{\Lambda} Q^{\mathbf{A} \Lambda}, & \omega_{\mathbf{A} \Lambda} Q^{\mathbf{B} \Lambda}=\omega_{\mathbf{A}}{ }^{\Lambda} Q^{\mathbf{B}}{ }_{\Lambda}, \\
\omega_{\mathbf{A} \Lambda} Q^{\mathbf{A}}{ }_{\Sigma}=\omega_{\mathbf{A} \Sigma} Q^{\mathbf{A}}{ }_{\Lambda}, & \omega_{\mathbf{A}}{ }^{\Lambda} Q^{\mathbf{A}}{ }_{\Sigma}=\omega_{\mathbf{A} \Sigma} Q^{\mathbf{A} \Lambda}, & \omega_{\mathbf{A}}{ }^{\Lambda} Q^{\mathbf{A} \Sigma}=\omega_{\mathbf{A}}{ }^{\Sigma} Q^{\mathbf{A} \Lambda} .
\end{array}
$$

To be more specific in terms of the conventional $H, \omega, Q$, and $R$ flux, using the definitions in Eq. (2.8), the compactly written six constraints in (2.10) turn out to represent five classes of Bianchi identities, which have a total of 11 constraints as collected in Table II.

Similarly, imposing the nilpotency of twisted differential operator $\mathcal{D}$ via $\mathcal{D}^{2} A_{p}=0$ where $A_{p}=\frac{1}{p !} X_{i_{1} \ldots i_{p}} d x^{1} \wedge$ $d x^{2} \ldots \wedge d x^{p}$, one gets the five sets of Bianchi identities along with an "extra constraint" in what we call the "standard formulation." These are given as follows [36,51,165]:
(I) $H_{m[\underline{i j}} \omega_{k l]}{ }^{m}=0$,
(II) $\omega_{[\underline{[i j}}^{m} \omega_{\underline{k}] m}{ }^{l}=Q_{[\underline{i}}^{l m} H_{\underline{j k}] m}$,
(III) $H_{i j m} R^{m k l}+\omega_{i j}{ }^{m} Q_{m}{ }^{k l}=4 Q_{[\underline{i}}{ }^{m[\underline{k}} \omega_{\underline{j}] m^{l]}}$,
(IV) $\left.Q_{m} \stackrel{[i j}{{ }^{i j}} Q_{l}^{\underline{k}] m}=\omega_{l m}{ }^{[i} R^{j k}\right] m$,
(V) $R^{m[\underline{i j}} Q_{m} \underline{k l]}=0$,
Constraint : $\frac{1}{6} H_{i j k} R^{i j k}+\frac{1}{2} \omega_{i j}{ }^{k} Q_{k}{ }^{i j}=0$. 
TABLE II. Bianchi identities of the cohomology formulation and their counting.

\begin{tabular}{|c|c|c|}
\hline Class & Bianchi identities of the cohomology formulation & Maximum no. of identities \\
\hline (I) & $H_{\Lambda} \omega_{A}{ }^{\Lambda}=H^{\Lambda} \omega_{A \Lambda}$ & $h^{1,1}$ \\
\hline \multirow[t]{2}{*}{ (II) } & $H_{\Lambda} Q^{A \Lambda}=H^{\Lambda} Q_{\Lambda}^{A}$ & $h^{1,1}$ \\
\hline & $\omega_{A}^{\Lambda} \omega_{B \Lambda}=\omega_{B}^{\Lambda} \omega_{A \Lambda}$ & $\frac{1}{2} h^{1,1}\left(h^{1,1}-1\right)$ \\
\hline \multirow[t]{4}{*}{ (III) } & $H^{\Lambda} R_{\Lambda}=H_{\Lambda} R^{\Lambda}$ & 1 \\
\hline & $\begin{aligned} \omega_{A}{ }^{\Lambda} Q^{B}{ }_{\Lambda \Sigma} & =\omega_{A \Lambda} Q^{B \Lambda} \\
R^{\Lambda} H^{\Sigma}+\omega_{\Lambda}{ }^{\Lambda} O^{A \Sigma} & =H^{\Lambda} R^{\Sigma}+O^{A \Lambda} \omega_{\Lambda} \Sigma\end{aligned}$ & $\begin{array}{c}\left(h^{1,1}\right)^{2} \\
1 h^{2,1}\left(h^{2,1}+1\right)\end{array}$ \\
\hline & $R_{\Lambda} H_{\Sigma}+\omega_{A \Lambda} Q^{A}{ }_{\Sigma}=H_{\Lambda} R_{\Sigma}+Q^{A}{ }_{\Lambda} \omega_{A \Sigma}$ & $\frac{1}{2} h^{2,1}\left(h^{2,1}+1\right)$ \\
\hline & $R_{\Lambda} H^{\Sigma}+\omega_{A \Lambda} Q^{A \Sigma}=H_{\Lambda} R^{\Sigma}+Q^{A}{ }_{\Lambda} \omega_{A}{ }^{\Sigma}$ & $\left(h^{2,1}+1\right)^{2}$ \\
\hline \multirow[t]{2}{*}{$(\mathbf{I V})$} & $Q^{A}{ }_{\Lambda} Q^{B \Lambda}=Q^{B}{ }_{\Lambda} Q^{A \Lambda}$ & $\frac{1}{2} h^{1,1}\left(h^{1,1}-1\right)$ \\
\hline & $R_{\Lambda} \omega_{A}^{\Lambda}=R^{\Lambda} \omega_{A \Lambda}$ & $h^{1,1}$ \\
\hline \multirow[t]{2}{*}{$(\mathbf{V})$} & $R_{\Lambda} Q^{A \Lambda}=R^{\Lambda} Q^{A}{ }_{\Lambda}$ & $h^{1,1}$ \\
\hline & Total & $\left(h^{1,1}+1\right)\left(2 h^{1,1}+1\right)+\left(h^{2,1}+1\right)\left(2 h^{2,1}+1\right)$ \\
\hline
\end{tabular}

Also let us mention that, for our current interest, we consider the fluxes to be constant parameters; however, for the nonconstant fluxes and in the presence of sources, these Bianchi identities in Eq. (2.11) are modified [48,49,166,167].

\section{SOLUTIONS OF BIANCHI IDENTITIES FOR $\mathbb{T}^{6} /\left(\mathbb{Z}_{3} \times \mathbb{Z}_{3}\right)$ SETUPS}

In this section, we discuss the Bianchi identities and their solutions for the $\mathcal{N}=2$ theory obtained in nongeometric compactifications using $\mathbb{T}^{6} /\left(\mathbb{Z}_{3} \times \mathbb{Z}_{3}\right)$ orbifold, and subsequently we will present them for the type IIA and type IIB orientifolds as well.

\section{A. Capturing the $\mathcal{N}=\mathbf{2}$ missing Bianchi identities}

Using the cohomology formulation Bianchi identities given in Table II, we find the following set of constraints for this rigid toroidal orbifold,

$$
\begin{aligned}
H_{0} \omega_{A}{ }^{0} & =H^{0} \omega_{A 0}, \quad H_{0} Q^{A 0}=H^{0} Q^{A}{ }_{0}, \quad \omega_{A 0} \omega_{B}{ }^{0}=\omega_{B 0} \omega_{A}{ }^{0}, \\
R_{0} Q^{A 0} & =R^{0} Q^{A}, \quad R_{0} \omega_{A}{ }^{0}=R^{0} \omega_{A 0}, \quad Q^{A 0} Q^{B}{ }_{0}=Q^{B 0} Q^{A}, \\
R_{0} H^{0} & =H_{0} R^{0}, \quad \omega_{A 0} Q^{A 0}=\omega_{A}{ }^{0} Q^{A}, \\
\omega_{A 0} Q^{B 0} & =\omega_{A}{ }^{0} Q^{B}, \quad \forall A, B=1,2,3 .
\end{aligned}
$$

As expected the total number of cohomology formulation identities is 29 , which follows from $\left(h^{1,1}+1\right)\left(2 h^{1,1}+\right.$ 1) $+\left(h^{2,1}+1\right)\left(2 h^{2,1}+1\right)$ as $h^{1,1}=3$ and $h^{2,1}=0$ for the present orbifold construction. While we place the explicit computations in the Appendix A, the various flux conversion relations from the standard formulation to the cohomology formulation are given as follows:

$$
\begin{array}{rlrl}
H_{0} & =H_{135}, & H^{0}=H_{246}, & \\
\omega_{10} & =-\omega_{46}{ }^{2}, & \omega_{20}=-\omega_{62}{ }^{4}, & \omega_{30}=-\omega_{24}{ }^{6}, \\
\omega_{1}{ }^{0} & =-\omega_{46}{ }^{1}, & \omega_{2}{ }^{0}=-\omega_{62}{ }^{3}, & \omega_{3}{ }^{0}=-\omega_{24}{ }^{5}, \\
Q^{1}{ }_{0} & =Q_{2}{ }^{45}, & Q^{2}{ }_{0}=Q_{4}{ }^{61}, & Q^{3}{ }_{0}=Q_{6}{ }^{23}, \\
Q^{10} & =-Q_{2}{ }^{46}, & Q^{20}=-Q_{4}{ }^{62}, & Q^{30}=-Q_{6}{ }^{24}, \\
R_{0} & =R^{246}, & R^{0}=-R^{135} .
\end{array}
$$

In order to capture the missing Bianchi identities, we consider the flux constraints of the standard formulation presented in Eq. (2.11). Subsequently, using the flux conversion relations given in Eq. (3.2) one can translate those standard Bianchi identities into cohomology form, which after some reshuffling can be presented by a set of flux constraints collected as follows:

$$
\begin{aligned}
& \text { (I) } H_{0} \omega_{A}{ }^{0}=H^{0} \omega_{A 0}, \quad \forall A=1,2,3 . \\
& \text { (II ) } H_{0} Q^{A 0}=H^{0} Q^{A}{ }_{0}, \quad \omega_{A 0} \omega_{B}{ }^{0}=\omega_{B 0} \omega_{A}{ }^{0} ; \quad \forall A=1,2,3 . \\
& \quad \omega_{A 0} \omega_{B 0}+\omega_{A 0} \omega_{B 0}=H_{0} Q^{C}{ }_{0}+H^{0} Q^{C 0} ; \quad A \neq B \neq C ; \\
& \text { (III I) } R_{0} H^{0}=H_{0} R^{0}, \quad \omega_{A 0} Q^{B 0}=\omega_{A}{ }^{0} Q^{B}{ }_{0}, \quad \forall A, B ; \\
& \quad H_{0} R_{0}+H^{0} R^{0}=\omega_{10} Q^{10}+\omega_{1}{ }^{0} Q^{1}{ }_{0}=\omega_{20} Q^{20}+\omega_{2}{ }^{0} Q^{2}{ }_{0}=\omega_{30} Q^{30}+\omega_{3}{ }^{0} Q^{3}{ }_{0}, \\
& \text { (IV) } R_{0} \omega_{A}{ }^{0}=R^{0} \omega_{A 0}, \quad Q^{A 0} Q^{B}{ }_{0}=Q^{B 0} Q^{A}{ }_{0} ; \quad \forall A=1,2,3 . \\
& \quad Q^{A}{ }_{0} Q^{B}{ }_{0}+Q^{A 0} Q^{B 0}=R_{0} \omega_{C 0}+R^{0} \omega_{C}{ }^{0} ; \quad A \neq B \neq C, \\
& \text { (V) } R_{0} Q^{A 0}=R^{0} Q^{A}{ }_{0} \quad \forall A=1,2,3 .
\end{aligned}
$$


In addition, the "extra constraint" presented for the standard formulation as given in Eq. (2.11) translates into the following cohomology form:

$$
R_{0} H^{0}+\omega_{A 0} Q^{A 0}=H_{0} R^{0}+\omega_{A}^{0} Q^{A} .
$$

Therefore comparing the constraints arising in the two formulations, we obtain the following nine what we call "missing" Bianchi identities,

$$
\begin{aligned}
\omega_{A 0} \omega_{B 0}+\omega_{A}{ }^{0} \omega_{B}{ }^{0} & =H_{0} Q^{C}{ }_{0}+H^{0} Q^{C 0}, \quad A \neq B \neq C ; \\
Q^{A}{ }_{0} Q^{B}{ }_{0}+Q^{A 0} Q^{B 0} & =R_{0} \omega_{C 0}+R^{0} \omega_{C}{ }^{0}, \quad A \neq B \neq C ; \\
H_{0} R_{0}+H^{0} R^{0} & =\omega_{10} Q^{1}{ }_{0}+\omega_{1}{ }^{0} Q^{10}=\omega_{20} Q^{2}{ }_{0}+\omega_{2}{ }^{0} Q^{20}=\omega_{30} Q^{3}{ }_{0}+\omega_{3}{ }^{0} Q^{30} .
\end{aligned}
$$

These identities certainly constrain the flux choice allowed by the usual cohomology formulation, and hence can have the potential to significantly affect any $\mathcal{N}=2$ phenomenological conclusion derived from the scalar potential. Moreover, we find that using the triple intersection numbers $\kappa_{A B C}$ such that $\kappa_{123}=1$, these missing identities can be expressed in the following manner:

$$
\begin{aligned}
& \left.\left.H_{(\underline{0}} Q^{A} \underline{0}\right)+H^{(0} Q^{A \underline{0})}=\frac{1}{2} \kappa_{A B C}^{-1} \omega_{B(\underline{0}} \omega_{C \underline{0})}+\frac{1}{2} \kappa_{A B C}^{-1} \omega_{B}{ }^{(0} \omega_{C} \underline{0}\right), \quad \forall A \in h^{1,1} ; \\
& \left.\left.3 H_{(\underline{0}} R_{\underline{0})}+3 H^{(\underline{0}} R^{0}\right)=\omega_{A(\underline{0}} Q^{A} \underline{0}\right)+\omega_{A}\left(\underline{0} Q^{A \underline{0})}\right. \text {; } \\
& \left.\left.\left.H_{(\underline{0}} R_{0}\right)+H^{(\underline{0}} R^{0}\right)=\omega_{A^{\prime}(\underline{0}} Q^{A^{\prime}} \underline{\underline{0}}\right)+\omega_{A^{\prime}}{ }^{\prime} \underline{0} Q^{\left.A^{\prime} \underline{0}\right)}, \quad \forall A^{\prime} \in h^{1,1} \quad \text { and } A^{\prime} \text { not summed over; } \\
& \left.\left.R_{(\underline{0}} \omega_{A \underline{0})}+R^{(\underline{0}} \omega_{A} \underline{0}\right)=\frac{1}{2} \kappa_{A B C} Q^{B}{ }_{(\underline{0}} Q^{C}{ }_{0}\right)+\frac{1}{2} \kappa_{A B C} Q^{B(\underline{0}} Q^{C \underline{0})}, \quad \forall A \in h^{1,1} .
\end{aligned}
$$

In the above, $\kappa_{A B C}^{-1}=1 / \kappa_{A B C}$ for fixed $A, B, C$ whenever $\kappa_{A B C} \neq 0$. It is important to mention that the above set of identities are well along the line with the conjectured form of the $\mathcal{N}=1$ missing Bianchi identities presented in [69].

\section{B. Bianchi identities for the type IIA orientifold}

For the type IIA orientifold, the various flux action inherited from the $\mathcal{N}=2$ theory can be given as follows:

$$
\begin{aligned}
& H \wedge \mathbf{1}=H_{K} \beta^{K}, \quad H \wedge \alpha_{K}=-H_{K} \Phi_{6}, \quad H \wedge \beta^{K}=0, \\
& \omega \triangleleft \nu_{a}=-\omega_{a K} \beta^{K}, \quad \omega \triangleleft \mu_{\alpha}=\hat{\omega}_{\alpha}^{K} \alpha_{K}, \quad \omega \triangleleft \alpha_{K}=-\omega_{a K} \tilde{\nu}^{a}, \quad \omega \triangleleft \beta^{K}=-\hat{\omega}_{\alpha}^{K} \tilde{\mu}^{\alpha}, \\
& Q \triangleright \tilde{\nu}^{a}=Q_{K}^{a} \beta^{K}, \quad Q \triangleright \tilde{\mu}^{\alpha}=-\hat{Q}^{\alpha K} \alpha_{K}, \quad Q \triangleright \alpha_{K}=-Q_{K}^{a} \nu_{a}, \quad Q \triangleright \beta^{K}=-\hat{Q}^{\alpha K} \mu_{\alpha}, \\
& R \cdot \Phi_{6}=-R_{K} \beta^{K}, \quad R \cdot \alpha_{K}=-R_{K} \mathbf{1}, \quad R \cdot \beta^{K}=0 .
\end{aligned}
$$

In addition, we get the following set of Bianchi identities in the cohomology formulation:

$$
\begin{aligned}
& H_{K} \hat{\omega}_{\alpha}{ }^{K}=0, \quad H_{K} \hat{Q}^{\alpha K}=0, \quad R_{K} \hat{Q}^{\alpha K}=0, \quad R_{K} \hat{\omega}_{\alpha}{ }^{K}=0, \\
& \omega_{a K} \hat{\omega}_{\alpha}{ }^{K}=0, \quad \omega_{a K} \hat{Q}^{\alpha K}=0, \quad Q^{a}{ }_{K} \hat{Q}^{\alpha K}=0, \quad \hat{\omega}_{\alpha}{ }^{K} Q^{a}{ }_{K}=0, \\
& \hat{\omega}_{\alpha}{ }^{[K} \hat{Q}^{\alpha J]}=0, \quad H_{[K} R_{J]}=\omega_{a[K} Q^{a}{ }_{J]},
\end{aligned}
$$

where the bracket [..] appearing in the last two identities denote the antisymmetrization of $J$ and $K$ indices.

The various fluxes that appear in the $\mathcal{N}=1$ type IIA orientifold framework are given, along with their respective conversion relations to the cohomology formulation, as follows:

$$
\begin{aligned}
& H_{0}=H_{135}, \quad \omega_{10}=-\omega_{46}{ }^{2}, \quad \omega_{20}=-\omega_{62}{ }^{4} \quad \omega_{30}=-\omega_{24}{ }^{6}, \\
& Q^{1}{ }_{0}=Q_{2}{ }^{45}, \quad Q^{2}{ }_{0}=Q_{4}{ }^{61}, \quad Q^{3}{ }_{0}=Q_{6}{ }^{23}, \quad R_{0}=R^{246} .
\end{aligned}
$$

Let us mention that for our type IIA setup with $\mathbb{T}^{6} /\left(\mathbb{Z}_{3} \times \mathbb{Z}_{3}\right)$ orientifold using the standard involution $\sigma_{I I A}: z^{i} \rightarrow-\bar{z}^{i}$, the even $(1,1)$ cohomology and its dual odd $(2,2)$ cohomology are trivial, i.e., $h_{+}^{1,1}=0$, and therefore all the "hatted" fluxes in Eq. (3.8) that are counted via $\alpha \in h_{+}^{1,1}$ are projected out. Such a situation provides a strong constraint on the cohomology formulation of the Bianchi identities as it suggests that all the Bianchi identities of the class $(\mathbf{I}),(\mathbf{I I}),(\mathbf{I V})$, and $(\mathbf{V})$ are identically trivial. In fact, the only Bianchi identities that could be nontrivial turns out to be the following one: 


$$
H_{[K} R_{J]}=\omega_{a[K} Q_{J]}^{a}
$$

However, for the case of frozen complex structure moduli, even the above identity is trivially satisfied. Thus, in our current type IIA construction, all the cohomology formulation identities are trivial. However translating the standard formulation identities using the conversion relations in Eq. (3.10), one ends up having the following status on the Bianchi identities,

$$
\begin{aligned}
& \text { (I) Trivial } \\
& \text { (II ) } \omega_{10} \omega_{20}=H_{0} Q^{3}{ }_{0}, \quad \omega_{20} \omega_{30}=H_{0} Q^{1}{ }_{0}, \quad \omega_{30} \omega_{10}=H_{0} Q^{2}{ }_{0} \text {, } \\
& \text { (I I I) } H_{0} R_{0}=\omega_{10} Q^{1}{ }_{0}=\omega_{20} Q^{2}{ }_{0}=\omega_{30} Q^{3}{ }_{0} \text {, } \\
& \text { (IV) } Q^{1}{ }_{0} Q^{2}{ }_{0}=R_{0} \omega_{30}, \quad Q^{2}{ }_{0} Q^{3}{ }_{0}=R_{0} \omega_{10}, \quad, \quad Q^{3}{ }_{0} Q^{1}{ }_{0}=R_{0} \omega_{20} \text {, } \\
& \text { (V) Trivial }
\end{aligned}
$$$$
\text { Trivial (extra constraint). }
$$

After looking at the index structure, it is clear that these identities correspond to the missing class, and do not arise from the list of cohomology identities. Moreover these can be also written in the following form:

$$
\begin{aligned}
& H_{(\underline{0}} Q^{a}{ }_{\underline{0})}=\frac{1}{2} \kappa_{a b c}^{-1} \omega_{b(\underline{0}} \omega_{c \underline{0})}, \quad \forall a: \hat{\kappa}_{a \alpha \beta}=0 ; \\
& 3 H_{(\underline{0}} R_{\underline{0})}=\omega_{a(\underline{0}} Q^{a}{ }_{\underline{0})}+\hat{\omega}_{\alpha}\left(\underline{0} \hat{Q}^{\alpha \underline{0})} ;\right. \\
& R_{(\underline{0}} \omega_{a \underline{0})}=\frac{1}{2} \kappa_{a b c} Q^{b}{ }_{(\underline{0}} Q^{c}{ }_{\underline{0})}, \quad \forall a: \hat{\kappa}_{a \alpha \beta}=0,
\end{aligned}
$$

which matches with the conjecture given in [69]. Here we used $\kappa_{a b c}^{-1}=1 / \kappa_{a b c}$ for fixed $a, b, c$ whenever $\kappa_{a b c}$ is nonzero. Recall that for the current toroidal model, the intersection numbers of type $\hat{\kappa}_{a \alpha \beta}$ are absent due to orientifold projection, and, for this reason, the flux components $\hat{\omega}_{\alpha}{ }^{0}$ and $\hat{Q}^{\alpha 0}$ are also projected out. The various possible solutions of these Bianchi identities presented in Eq. (3.12) can be expressed in the following eight classes:

$$
\begin{aligned}
& \text { S1: } \omega_{10}=\omega_{20}=\omega_{30}=0, \quad Q^{1}{ }_{0}=Q^{2}{ }_{0}=Q^{3}{ }_{0}=0, \quad R_{0}=0 ; \\
& \text { S2: } H_{0}=0, \quad \omega_{10}=\omega_{20}=\omega_{30}=0, \quad Q^{1}{ }_{0}=Q^{2}{ }_{0}=Q^{3}{ }_{0}=0 \text {; } \\
& \text { S3: } R_{0}=0, \quad Q^{1}{ }_{0}=Q^{2}{ }_{0}=Q^{3}{ }_{0}=0, \quad \omega_{20}=0=\omega_{30}, \quad \omega_{10} \neq 0 ; \\
& R_{0}=0, \quad Q^{1}{ }_{0}=Q^{2}{ }_{0}=Q^{3}{ }_{0}=0, \quad \omega_{10}=0=\omega_{30}, \quad \omega_{20} \neq 0 ; \\
& R_{0}=0, \quad Q^{1}{ }_{0}=Q^{2}{ }_{0}=Q^{3}{ }_{0}=0, \quad \omega_{10}=0=\omega_{20}, \quad \omega_{30} \neq 0 ; \\
& \text { S4: } H_{0}=0, \quad \omega_{10}=\omega_{20}=\omega_{30}=0, \quad Q^{2}{ }_{0}=0=Q^{3}{ }_{0}, \quad Q^{1}{ }_{0} \neq 0 \text {; } \\
& H_{0}=0, \quad \omega_{10}=\omega_{20}=\omega_{30}=0, \quad Q^{1}{ }_{0}=0=Q^{3}{ }_{0}, \quad Q^{2} \neq 0 ; \\
& H_{0}=0, \quad \omega_{10}=\omega_{20}=\omega_{30}=0, \quad Q^{1}{ }_{0}=0=Q^{2}{ }_{0}, \quad Q^{3} \neq 0 ; \\
& \text { S5: } H_{0}=0, \quad \omega_{20}=0=\omega_{30}, \quad Q^{1}{ }_{0}=0=Q^{2}{ }_{0}, \quad R_{0}=0, \quad \omega_{10} \neq 0, \quad Q^{3}{ }_{0} \neq 0 \text {; } \\
& H_{0}=0, \quad \omega_{10}=0=\omega_{30}, \quad Q^{1}{ }_{0}=0=Q^{2}{ }_{0}, \quad R_{0}=0, \quad \omega_{20} \neq 0, \quad Q^{3} \neq 0 ; \\
& H_{0}=0, \quad \omega_{20}=0=\omega_{30}, \quad Q^{1}{ }_{0}=0=Q^{3}{ }_{0}, \quad R_{0}=0, \quad \omega_{10} \neq 0, \quad Q^{2} \neq 0 ; \\
& H_{0}=0, \quad \omega_{10}=0=\omega_{20}, \quad Q^{1}{ }_{0}=0=Q^{3}{ }_{0}, \quad R_{0}=0, \quad \omega_{30} \neq 0, \quad Q^{2}{ }_{0} \neq 0 ; \\
& H_{0}=0, \quad \omega_{10}=0=\omega_{20}, \quad Q^{2}{ }_{0}=0=Q^{3}{ }_{0}, \quad R_{0}=0, \quad \omega_{30} \neq 0, \quad Q^{1} \neq 0 ; \\
& H_{0}=0, \quad \omega_{10}=0=\omega_{30}, \quad Q^{2}{ }_{0}=0=Q^{3}, \quad R_{0}=0, \quad \omega_{20} \neq 0, \quad Q^{1} \neq 0 \text {; }
\end{aligned}
$$




$$
\begin{aligned}
& \text { S6: } H_{0}=0, \quad Q^{1}=0, \quad \omega_{20}=0=\omega_{30}, \quad R_{0}=\frac{Q^{2}{ }_{0} Q^{3}{ }_{0}}{\omega_{10}}, \quad \omega_{10} \neq 0, \quad Q^{2}{ }_{0} Q^{3}{ }_{0} \neq 0 \text {; } \\
& H_{0}=0, \quad Q^{2}{ }_{0}=0, \quad \omega_{10}=0=\omega_{30}, \quad R_{0}=\frac{Q^{1}{ }_{0} Q^{3}{ }_{0}}{\omega_{20}}, \quad \omega_{20} \neq 0, \quad Q^{1}{ }_{0} Q^{3}{ }_{0} \neq 0 ; \\
& H_{0}=0, \quad Q^{3}{ }_{0}=0, \quad \omega_{10}=0=\omega_{20}, \quad R_{0}=\frac{Q^{1}{ }_{0} Q^{2}{ }_{0}}{\omega_{30}}, \quad \omega_{30} \neq 0, \quad Q^{1}{ }_{0} Q^{2}{ }_{0} \neq 0 ;
\end{aligned}
$$

S7: $R_{0}=0, \quad \omega_{10}=0, \quad Q^{2}{ }_{0}=0=Q^{3}{ }_{0}, \quad H_{0}=\frac{\omega_{20} \omega_{30}}{Q^{1}{ }_{0}}, \quad Q^{1}{ }_{0} \neq 0, \quad \omega_{20} \omega_{30} \neq 0 ;$

$$
\begin{aligned}
& R_{0}=0, \quad \omega_{20}=0, \quad Q^{1}{ }_{0}=0=Q^{3}{ }_{0}, \quad H_{0}=\frac{\omega_{10} \omega_{30}}{Q^{2}{ }_{0}}, \quad Q^{2}{ }_{0} \neq 0, \quad \omega_{10} \omega_{30} \neq 0 ; \\
& R_{0}=0, \quad \omega_{30}=0, \quad Q^{1}{ }_{0}=0=Q^{2}{ }_{0}, \quad H_{0}=\frac{\omega_{10} \omega_{20}}{Q^{3}{ }_{0}}, \quad Q^{3}{ }_{0} \neq 0, \quad \omega_{20} \omega_{10} \neq 0 ;
\end{aligned}
$$$$
\text { S8: }\left\{Q^{1}{ }_{0} \neq 0, \quad Q^{2}{ }_{0} \neq 0, \quad Q^{3}{ }_{0} \neq 0, \quad \omega_{10}=\frac{\omega_{30} Q^{3}{ }_{0}}{Q^{1}{ }_{0}}, \quad \omega_{20}=\frac{\omega_{30} Q^{3}{ }_{0}}{Q^{2}{ }_{0}}, \quad \omega_{30} \neq 0,\right.
$$$$
\left.H_{0}=\frac{\omega_{10} \omega_{20}}{Q^{3}{ }_{0}}, \quad R_{0}=\frac{Q^{1}{ }_{0} Q^{2}{ }_{0}}{\omega_{30}}\right\}
$$

where in the above solutions if some flux components are not mentioned then it means that they are not constrained in the given solution of identities. We will utilize these flux constraints for exploring the de Sitter (no-go) scenarios in one of the upcoming sections.

\section{Lessons from the missing Bianchi identities in the type IIB orientifold}

The various fluxes that appear in the $\mathcal{N}=1$ type IIB orientifold framework are given, along with their respective conversion relations to the cohomology formulation, as follows:

$$
\begin{aligned}
& H_{0}=H_{135}, \quad Q^{1}{ }_{0}=Q_{2}{ }^{45}, \quad Q^{2}{ }_{0}=Q_{4}{ }^{61}, \quad Q^{3}{ }_{0}=Q_{6}{ }^{23}, \\
& H^{0}=H_{246}, \quad Q^{10}=-Q_{2}{ }^{46}, \quad Q^{20}=-Q_{4}{ }^{62}, \quad Q^{30}=-Q_{6}{ }^{24} \text {. }
\end{aligned}
$$

Thus we have eight independent NS-NS flux components that survive the orbifold twist, and they are further constrained by the set of Bianchi identities, which turns out to be of the following form:

(I) Trivial

(I I) $H_{0} Q^{10}=H^{0} Q^{1}{ }_{0}, \quad H_{0} Q^{20}=H^{0} Q^{2}{ }_{0}, \quad H_{0} Q^{30}=H^{0} Q^{3}{ }_{0}$,

$$
H_{0} Q^{1}{ }_{0}+H^{0} Q^{10}=0, \quad H_{0} Q^{2}{ }_{0}+H^{0} Q^{20}=0, \quad H_{0} Q^{3}{ }_{0}+H^{0} Q^{30}=0,
$$

(III) Trivial

(IV) $Q^{10} Q^{2}{ }_{0}=Q^{20} Q^{1}{ }_{0}, \quad Q^{10} Q^{3}{ }_{0}=Q^{30} Q^{1}{ }_{0}, \quad Q^{20} Q^{3}{ }_{0}=Q^{30} Q^{2}{ }_{0}$,

$$
Q^{1}{ }_{0} Q^{2}{ }_{0}+Q^{10} Q^{20}=0, \quad Q^{2}{ }_{0} Q^{3}{ }_{0}+Q^{2} Q^{30}=0, \quad Q^{1}{ }_{0} Q^{3}{ }_{0}+Q^{10} Q^{30}=0,
$$

(V) Trivial

Trivial (extra constraint).

Let us first analyze the cohomology formulation identities pretending that those are the only ones to worry about. In this regard, there are six such flux constraints that arise from the two classes of identities, namely $H^{\Lambda} Q^{A}{ }_{\Lambda}=H_{\Lambda} Q^{A \Lambda}$ and $Q^{A \Lambda} Q^{B}{ }_{\Lambda}=Q^{A}{ }_{\Lambda} Q^{B \Lambda}$. These can be explicitly given as follows:

$$
\begin{aligned}
& H_{0} Q^{10}=H^{0} Q^{1}{ }_{0}, \quad H_{0} Q^{20}=H^{0} Q^{2}{ }_{0}, \quad H_{0} Q^{30}=H^{0} Q^{3}{ }_{0}, \\
& Q^{10} Q^{2}{ }_{0}=Q^{20} Q^{1}{ }_{0}, \quad Q^{10} Q^{3}{ }_{0}=Q^{30} Q^{1}{ }_{0}, \quad Q^{20} Q^{3}{ }_{0}=Q^{30} Q^{2}{ }_{0} \text {. }
\end{aligned}
$$


These constraint are indeed a part of the standard set of Bianchi identities computed for this model as given in Eq. (3.15). Assuming the case for $H \neq 0$ and $Q \neq 0$, i.e., when both the kinds of fluxes have at least one nonzero component, we find that there are four classes of solutions of the above set of Bianchi identities in Eq. (3.16), which can be given as follows:

$$
\begin{aligned}
& \text { (S1): } H^{0}=Q^{10}=Q^{20}=Q^{30}=0 \text {, } \\
& (\mathbf{S 2}): Q^{20}=Q^{30}=Q^{2}{ }_{0}=Q^{3}{ }_{0}=0 \text {, } \\
& Q^{10} \neq 0, \quad H_{0}=\frac{Q^{1}{ }_{0} H^{0}}{Q^{10}}, \\
& (\mathbf{S 3}): Q^{30}=Q^{3}{ }_{0}=0, \quad Q^{20} \neq 0, \\
& Q^{1}{ }_{0}=\frac{Q^{10} Q^{2}{ }_{0}}{Q^{20}}, \quad H_{0}=\frac{Q^{2}{ }_{0} H^{0}}{Q^{20}}, \\
& (\mathbf{S 4}): Q^{30} \neq 0, \quad Q^{1}{ }_{0}=\frac{Q^{10} Q^{3}{ }_{0}}{Q^{30}}, \\
& Q^{2}{ }_{0}=\frac{Q^{20} Q^{3} 0}{Q^{30}}, \quad H_{0}=\frac{Q^{3} H_{0}^{0}}{Q^{30}} .
\end{aligned}
$$

Now we can observe that if one does not consider the set of missing identities, there are indeed some possible nontrivial solutions. In fact the solution (S1) is a quite popular one in type IIB orientifold compactifications with nongeometric fluxes as the cohomology formulation identities given in Eq. (3.16) can be trivially satisfied by switching off half of the fluxes, either with the lower or with the upper $h^{2,1}$ indices $[28,51,68]$.

However, imposing the additional (missing) identities from the full set of constraints given in Eq. (3.15) leads to vanishing of the $Q$ flux whenever we assume that $H$ flux has at least one nonzero component in order to stabilize the axion dilaton $(\tau)$, which is a universal chiral variable in type IIB setting. This observation should be also crucial for the recent phenomenological studies made for the case of frozen complex structure moduli, as these are the simplest possible setups which could have some nontrivial applications for moduli stabilization and breaking of the noscale structure using nongeometric fluxes. So the main observation or the lesson which we get after analyzing this type IIB compactification on the orientifold of a $\mathbb{T}^{6} /\left(\mathbb{Z}_{3} \times \mathbb{Z}_{3}\right)$ orbifold is the fact that $H \neq 0$ implies $Q=0$; i.e., no nongeometric flux can be turned on in this rigid orientifold without switching off the NS-NS threeform flux $H_{3}$. This observation is apparently quite strong and a peculiar one in its own.

In that regard, let us also consider even a simpler case of the isotropic limit for the current example. This leads to the following identifications of the flux components,

$$
\begin{array}{rlrl}
H_{0} & =h_{0}, \quad H^{0} & =\tilde{h}^{0}, & Q^{1}{ }_{0}=Q^{2}{ }_{0}=Q^{3}{ }_{0}=q^{1}{ }_{0}, \\
Q^{10} & =Q^{20}=Q^{30}=\tilde{q}^{10},
\end{array}
$$

which are constrained by the following three NS-NS Bianchi identities,

$$
\begin{aligned}
& h_{0} \tilde{q}^{10}=\tilde{h}^{0} q_{0}^{1}, \quad h_{0} q^{1}{ }_{0}+\tilde{h}^{0} \tilde{q}^{10}=0, \\
& \left(q^{1}{ }_{0}\right)^{2}+\left(\tilde{q}^{10}\right)^{2}=0
\end{aligned}
$$

Note that only the first identity corresponds to the second formulation while the later two are the missing ones. It is now obvious that setting $\tilde{h}^{0}=0=\tilde{q}^{10}$, which are usually sufficient to satisfy the first identity, does not trivially satisfy the remaining two, and in fact leads to vanishing of $Q$ flux. On these lines, let us consider the following superpotential, which can be generically written for a setting (beyond the toroidal example such as Calabi Yau space), having the frozen complex structure moduli and a single Kähler modulus $T$,

$W=\left(f_{0}+\tau h_{0}+T q_{0}^{1}\right)+i\left(\tilde{f}^{0}+\tau \tilde{h}^{0}+T \tilde{q}^{10}\right)$,

in which the fluxes need to respect the single Bianchi identity $\tilde{h}^{0} q^{1}{ }_{0}=h_{0} \tilde{q}^{10}$ as known from the cohomology formulation. Therefore, for studying the phenomenology, what one usually does is to set $\tilde{h}^{0}=0=\tilde{q}^{10}$, which leads to the well-studied superpotential given as follows $[25,26,29,159]$ :

$$
W=f+\tau h_{0}+T q_{0}^{1}+i \tilde{f}^{0},
$$

and in addition to that one has to impose the RR tadpole cancellation conditions as well. In this case, the no-scale structure is broken by the nongeometric $\left(q_{0}^{1}\right)$ flux fixing the $T$ modulus, and results in a set of anti-de Sitter vacua, which would disappear if the missing Bianchi identities anticipated from the explicit computations for the rigid toroidal example continue to hold for the case of the rigid Calabi Yau orientifolds. However, one still needs to check if the missing identities we have anticipated to hold beyond the toroidal cases are indeed true for the generic rigid Calabi Yau compactifications.

With the reasons/observations elaborated as above, for the current article, we will not consider the study of nongeometric type IIB scalar potentials arising in the rigid orientifold flux compactifications, though the interested readers can read off the scalar potential for rigid compactifications from the generic results in $[56,60]$.

\section{TYPE IIA de Sitter NO-GO SCENARIOS}

The nongeometric type IIA scalar potential has been generically computed in [57], and we review the relevant pieces of information in the Appendix B. Given that the 
explicit expressions of the scalar potential in terms of various moduli and axions are known, we are not only in a position to rederive the various previously proposed no-go theorems regarding the de Sitter/inflationary realizations, but also we can explore more such conditions in a given specific setting with $\mathbb{T}^{6} /\left(\mathbb{Z}_{3} \times \mathbb{Z}_{3}\right)$ orientifolds as we plan to present in this section.

\section{A. Two known no-go conditions without nongeometric fluxes}

To begin with, using our generic results for the type IIA scalar potential we will rederive the de Sitter no-go conditions for two particular models which have been presented in [71,79,81-83]. The first no-go condition corresponds to the case of having no geometric and nongeometric fluxes [71,79], while the second case includes geometric flux but does not include the nongeometric flux [81-83].

In order to explore the possible de Sitter no-go scenarios, let us begin with introducing the notations of [82] by using the following redefinitions:

$$
\mathcal{V}=\rho^{3}, \quad t^{a}=\rho \gamma^{a}, \quad \kappa_{a b c} \gamma^{a} \gamma^{b} \gamma^{c}=6,
$$

where $\rho$ measures the overall volume of the internal manifold while $\gamma^{a}$ s correspond to the angular Kähler moduli. For invoking the "swamplandlike inequalities," we focus only on the dynamics in the volume-dilaton $(\rho-D)$ plane of the moduli space and ignore the other moduli dependences that otherwise would heavily complicate the setup. Given that the scalar potential can be expressed in terms of polynomials in $\rho$ and $e^{D}$ variables, this effective "two-field scenario" will not only keep the setup simple but will also impose quite interesting generic restrictions on the existence of de Sitter vacua. Being simple, this two-field model has been often found to be attractive and studying this model can also provide the "large volume" and "weak coupling" insights relevant for moduli stabilization and the other phenomenological aspects. However, our current aim is limited to study these models in the light of swampland inequalities given in Eqs. (1.1) and (1.2).

Using the new redefinitions in Eq. (4.1), the generic type IIA scalar potential pieces given in Eqs. (B7)-(B8) can be rewritten in the following manner:

$$
\begin{aligned}
V & =V_{h}+V_{\omega}+V_{q}+V_{r}+V_{f_{0}}+V_{f_{2}}+V_{f_{4}}+V_{f_{6}}+V_{\mathrm{loc}} ; \\
V_{h} & =\frac{e^{2 D}}{\rho^{3}} A_{h}, \quad V_{\omega}=\frac{e^{2 D}}{\rho} A_{\omega}, \quad V_{q}=e^{2 D} \rho A_{q}, \quad V_{r}=e^{2 D} \rho^{3} A_{r}, \\
V_{f_{0}} & =e^{4 D} \rho^{3} A_{f_{0}}, \quad V_{f_{2}}=e^{4 D} \rho A_{f_{2}}, \quad V_{f_{4}}=\frac{e^{4 D}}{\rho} A_{f_{4}}, \quad V_{f_{6}}=\frac{e^{4 D}}{\rho^{3}} A_{f_{6}}, \\
V_{\mathrm{loc}} & =e^{3 D} A_{\mathrm{loc}},
\end{aligned}
$$

where the various flux/moduli dependent quantities $A_{i} \mathrm{~s}$ are explicitly given by the following expressions:

$$
\begin{aligned}
A_{h}= & \frac{1}{2} \mathbb{I}_{I} \mathcal{M}^{I J} \mathbb{U}_{J}, \\
A_{\omega}= & \frac{1}{2}\left[\mho_{a I} \mathcal{M}^{I J} \mho_{b J} \gamma^{a} \gamma^{b}+4 \mho_{a I} \mho_{b J} \mathcal{X}^{I} \mathcal{X}^{J}\left(\tilde{K}^{a b}-\gamma^{a} \gamma^{b}\right)\right. \\
& \left.\quad-2\left(\mathbb{U}_{I} \mathcal{M}^{I J} \mathbb{Q}^{a}{ }_{J}-4 \mathbb{U}_{I} \mathcal{X}^{I} \mathcal{X}^{J} \mathbb{Q}^{a}{ }_{J}\right) \tilde{\sigma}_{a}+4 \mathcal{F}_{I} \mathcal{F}_{J} \hat{\mho}_{\alpha}{ }^{I}\left(\hat{\kappa}_{a \alpha \beta} \gamma^{a}\right)^{-1} \hat{\mho}_{\beta}{ }^{J}\right], \\
A_{q}= & \frac{1}{2}\left[\mathbb{Q}^{a}{ }_{I} \mathcal{M}^{I J} \mathbb{Q}^{b}{ }_{J} \tilde{\sigma}_{a} \tilde{\sigma}_{b}+4 \mathbb{Q}^{a}{ }_{I} \mathbb{Q}^{b}{ }_{J} \mathcal{X}^{I} \mathcal{X}^{J}\left(\tilde{K}_{a b}-\tilde{\sigma}_{a} \tilde{\sigma}_{b}\right)\right. \\
& \left.-2\left(\mathbb{R}_{I} \mathcal{M}^{I J} \mho_{a J}-4 \mathbb{R}_{I} \mathcal{X}^{I} \mathcal{X}^{J} \mho_{a J}\right) \gamma^{a}+4 \mathcal{F}_{I} \mathcal{F}_{J} \hat{\mathbb{Q}}^{\alpha I}\left(\hat{\kappa}_{a \alpha \beta} \gamma^{a}\right) \hat{\mathbb{Q}}^{\beta J}\right], \\
A_{r}= & \frac{1}{2} \mathbb{R}_{I} \mathcal{M}^{I J} \mathbb{R}_{J}, \\
A_{f_{0}}= & \frac{1}{2}\left(\mathbb{G}_{0}\right)^{2}, \quad A_{f_{2}}=\frac{1}{2} \mathbb{G}^{a} \tilde{K}_{a b} \mathbb{G}^{b}, \quad A_{f_{4}}=\frac{1}{2} \mathbb{G}_{a} \tilde{K}^{a b} \mathbb{G}_{b}, \quad A_{f_{6}}=\frac{1}{2}\left(\mathbb{G}^{0}\right)^{2}, \\
A_{\mathrm{loc}}= & -2\left(\mathbb{Q}_{K} \mathbb{G}_{0}-\mho_{a K} \mathbb{G}^{a}+\mathbb{Q}^{a}{ }_{K} \mathbb{G}_{a}-\mathbb{R}_{K} \mathbb{G}^{0}\right) \mathcal{X}^{K} .
\end{aligned}
$$

In the above collection, the axionic flux quantities $\left\{\mathbb{G}_{I}, \mho_{a I}, \mathbb{Q}^{a}{ }_{I}, \mathbb{R}_{I}\right\}$ and $\left\{\mathbb{G}_{0}, \mathbb{G}^{a}, \mathbb{G}_{a}, \mathbb{G}^{0}\right\}$ are defined in Eq. (B4) of the Appendix. Also note that, in the above collection $A_{i} \mathrm{~s}$, there are quantities involving only the angular Kähler moduli and not the $\rho$ modulus, as they have been obtained after extracting the volume modulus via using $t^{a}=\rho \gamma^{a}$. Similarly the moduli space metric and its inverse can be given after extracting the powers of $\rho$ in the following manner: 


$$
\begin{aligned}
\tilde{\sigma}_{a} & =\frac{1}{2} \kappa_{a b c} \gamma^{b} \gamma^{c}, \quad \tilde{K}^{a b}=\frac{1}{2} \gamma^{a} \gamma^{b}-\left(\kappa_{a b c} \gamma^{c}\right)^{-1}, \\
\tilde{K}_{a b} & =\tilde{\sigma}_{a} \tilde{\sigma}_{b}-\kappa_{a b c} \gamma^{c} .
\end{aligned}
$$

Note that none of the above quantities explicitly involve the $\rho$ modulus. One can easily convince that our scalar potential in Eqs. (4.2)-(4.3) reduces into the ones presented in [82,83] when the nongeometric $Q$ and $R$ fluxes are turned off but the geometric flux contributions are present; for example, see Eq. (2.34) of [82] and Eq. (3.13) of [83] where apart from the nongeometric fluxes, the $D$-term geometric fluxes $\hat{\omega}_{\alpha}{ }^{I}$ are also switched off.

In the context of extending the models with de Sitter solution to building inflationary scenarios, let us make a side remark that using the new basis of moduli through the relation given in Eq. (4.1), the slow-roll parameter $\epsilon_{V}$ can be simplified to satisfy the following bound [82]:

$$
\epsilon_{V} \geq \frac{1}{V^{2}}\left[\frac{\rho^{2}}{3}\left(\frac{\partial V}{\partial \rho}\right)^{2}+\frac{1}{4}\left(\frac{\partial V}{\partial D}\right)^{2}\right] .
$$

In fact, it has been shown in [82] that the slow-roll parameter $\epsilon_{V}$ can be expressed in terms of six non-negative pieces including the two of the above Eq. (4.5) which corresponds to derivatives of $\rho$ and $D$ moduli. So if there exists a set of parameters $\{\alpha>0, \beta>0\}$ such that one has an inequality of the form: $\left(\alpha \partial_{D} V-\rho \partial_{\rho} V\right) \geq \beta V$, then the generic extremum cannot be a de Sitter one because for this case $\partial_{D} V=0=\partial_{\rho} V$ would imply $V_{\text {ext }} \leq 0$. Therefore, finding such what we call "swamplandlike inequalities," namely $\left(\alpha \partial_{D} V-\rho \partial_{\rho} V\right) \geq \beta V$ for some positive quantities $\alpha \simeq \mathcal{O}(1)$ and $\beta \simeq \mathcal{O}(1)$ helps in determining the sign of potential at the extremum. Moreover, if such an inequality exists, using Eq. (4.5), one can convince that it leads to the following lower bound on the slow-roll parameter,

$$
\begin{aligned}
\epsilon_{V} \geq & V^{-2}\left[\frac{\rho^{2}}{3}\left(\partial_{\rho} V\right)^{2}+\frac{1}{4}\left(\partial_{D} V\right)^{2}\right], \\
= & \frac{V^{-2}}{3+4 \alpha^{2}}\left[\left(\alpha\left(\partial_{D} V\right)-\rho\left(\partial_{\rho} V\right)\right)^{2}\right. \\
& \left.+\frac{1}{12}\left(3\left(\partial_{D} V\right)+4 \alpha \rho\left(\partial_{\rho} V\right)\right)^{2}\right], \\
\geq & \frac{\beta^{2}}{3+4 \alpha^{2}} .
\end{aligned}
$$

This relation can be used for reading off the lower bound on $\epsilon_{V}$ parameter in several models as we will see in the upcoming subsections.

\section{NoGo-1: Without (non)geometric flux, i.e., $V_{\omega}=V_{q}=V_{r}=0$}

In the absence of any geometric and nongeometric fluxes, the scalar potential takes the following form

$$
V=V_{h}+V_{f_{0}}+V_{f_{2}}+V_{f_{4}}+V_{f_{6}}+V_{\mathrm{loc}}
$$

Recall that the generic expressions for the various scalar potential pieces such as $V_{h}, V_{\text {HI円 }}$ etc. are presented through Eqs. (B2), (B4), (B7), and (B8) of Appendix B. Here in this particular setting the term $V_{h}$ arises from $V_{\text {Ш甘円 }}$ after setting the $\omega, Q$, and $R$ fluxes to zero. Similarly, the RR potential terms $V_{f_{p}}$, for $p=0,2,4,6$, arise from their respective pieces after setting the $\omega, Q$, and $R$ fluxes to zero. This leads to the following form of the scalar potential pieces,

$V_{h}=\frac{e^{2 D}}{\rho^{3}} A_{h}, \quad V_{\mathrm{loc}}=e^{3 D} A_{\mathrm{loc}}, \quad V_{f_{p}}=\frac{e^{4 D}}{\rho^{(p-3)}} A_{f_{p}}$,

where $A_{h}$ and $A_{f_{p}}$ are flux dependent non-negative quantities that do not depend on the volume moduli $\rho$ and the dilaton $D$, while the local piece $A_{\mathrm{loc}}$ can a priori have any sign, however for avoiding the runaway in the dilatonic direction $D$, one needs $A_{\text {loc }}<0$. Now it is easy to observe that the following relations hold,

$$
\begin{aligned}
\partial_{D} V_{h} & =2 V_{h}, \quad \partial_{D} V_{\mathrm{loc}}=3 V_{\mathrm{loc}}, \\
\partial_{D} V_{f_{p}} & =4 V_{f_{p}}, \quad \forall p \in\{0,2,4,6\}, \\
\partial_{\rho} V_{h} & =-\frac{3}{\rho} V_{h}, \quad \partial_{\rho} V_{\mathrm{loc}}=0, \\
\partial_{\rho} V_{f_{p}} & =\frac{3-p}{\rho} V_{f_{p}}, \quad \forall p \in\{0,2,4,6\} .
\end{aligned}
$$

Using these partial derivatives results in the following inequality:

$$
3 \partial_{D} V-\rho \partial_{\rho} V=9 V+\sum_{p=2,4,6} p V_{f_{p}}>9 V
$$

Finding this information is very crucial for two reasons. First, it immediately shows that at the extremum in the two modulus $(\rho, D)$ plane, the scalar potential cannot be positive as one has

$$
V_{\mathrm{ext}}=-\frac{1}{9} \sum_{p=2,4,6} p V_{f_{p}}
$$

which therefore forbids de Sitter solution in this minimal setting. Second, using the Eq. (4.5), the inequality also leads to the following lower bound on the $\epsilon_{V}$ parameter,

$$
\epsilon_{V} \geq V^{-2}\left[\frac{\rho^{2}}{3}\left(\partial_{\rho} V\right)^{2}+\frac{1}{4}\left(\partial_{D} V\right)^{2}\right] \geq \frac{27}{13},
$$

which forbids any inflationary realization in this model. 
2. NoGo-2: Without nongeometric flux, i.e., $V_{q}=0=V_{r}$

The previous framework is very minimal and now we plan to include more ingredients to look for the possibility of avoiding the de Sitter no-go condition. After including the so-called geometric flux $\omega$, and still having no nongeometric fluxes, namely for the case when $Q=0=R$, the scalar potential takes the following form, ${ }^{1}$

$V=V_{h}+V_{\omega}+V_{f_{0}}+V_{f_{2}}+V_{f_{4}}+V_{f_{6}}+V_{\mathrm{loc}}$,

where as before $V_{h}$ arises from $V_{\text {ШШ }}$ but now only setting the $Q$ and $R$ fluxes to zero. Similarly, the RR potential terms $V_{f_{p}}$, for $p=0,2,4,6$, arise from their respective pieces after setting the $Q$ and $R$ fluxes to zero. In addition, now we have the $V_{\omega}$ piece, which can be generically sourced from both the $F$-term as well as the $D$-term contributions as seen from Eq. (B8). Let us recall here again that we are only setting the $Q$ and $R$ fluxes to zero, and not the geometric flux, and therefore $V_{h}$ will involve geometric flux contributions through the "generalized flux orbits" we have defined in Eq. (B4) to compactly write down the scalar potential. This is the case for the pieces $V_{f_{p}} \mathrm{~s}$ and the local piece $V_{\text {loc }}$, which involve the flux orbits as defined in Eq. (B4). With this in mind, we arrive at the following form of the scalar potential from our generic results,

$$
\begin{aligned}
V_{h} & =\frac{e^{2 D}}{\rho^{3}} A_{h}, & V_{\omega}=\frac{e^{2 D}}{\rho} A_{\omega}, \\
V_{\mathrm{loc}} & =e^{3 D} A_{D 6}, & V_{f_{p}}=\frac{e^{4 D}}{\rho^{(p-3)}} A_{f_{p}},
\end{aligned}
$$

where, as can be directly read off from the collection given in Eq. (4.3), one finds that $A_{h}, A_{\omega}, A_{\mathrm{loc}}$, and $A_{f_{p}}$ are flux dependent quantities that do not depend on the volume moduli $\rho$ and the dilaton $D$. Further, it is true that, except the term $A_{\omega}$ and the local term $A_{\text {loc }}$, all the remaining ones are guaranteed to be positive semidefinite. Although a couple of pieces within $A_{\omega}$ can be guaranteed to be positive semidefinite (e.g., the one coming from the $D$-term contributions), but the overall sign of $A_{\omega}$ cannot be a priori fixed. Moreover, all the terms have positive powers of $e^{D}$, and therefore to avoid a runaway in that direction one needs $A_{\text {loc }}<0$ in this case. Now it is easy to observe that

$$
\begin{array}{rlrl}
\partial_{D} V_{h} & =2 V_{h}, & \partial_{D} V_{\omega}=2 V_{\omega}, & \partial_{D} V_{\mathrm{loc}}=3 V_{\mathrm{loc}}, \\
\partial_{\rho} V_{h} & =-\frac{3}{\rho} V_{h}, & \partial_{\rho} V_{\omega}=-\frac{1}{\rho} V_{\omega}, & \partial_{\rho} V_{\mathrm{loc}}=0, \\
\partial_{D} V_{f_{p}} & =4 V_{f_{p}}, & \partial_{\rho} V_{f_{p}}=\frac{3-p}{\rho} V_{f_{p}} ; \quad \forall p \in\{0,2,4,6\} .
\end{array}
$$

\footnotetext{
${ }^{1}$ Recall that the generic expressions for the various scalar potential pieces such as $V_{h}, V_{\text {ㅁ® }}$ etc. are presented in Eqs. (B2), (B4), (B7), and (B8) of Appendix B.
}

Using these partial derivatives results in the following relation:

$\partial_{D} V-\rho \partial_{\rho} V=3 V+2 V_{h}+2 V_{f_{4}}+4 V_{f_{6}}-2 V_{f_{0}}$.

This presents an interesting fact. In the absence of Romans mass term, i.e., for $V_{f_{0}}=0$, one arrives at the following relations,

$\partial_{D} V-\rho \partial_{\rho} V=3 V+2 V_{h}+2 V_{f_{4}}+4 V_{f_{6}} \geq 3 V$,

which not only forbids the existence of de Sitter solution but also leads to the following lower bound on the $\epsilon_{V}$ parameter using Eq. (4.5),

$$
\epsilon_{V} \geq V^{-2}\left[\frac{\rho^{2}}{3}\left(\partial_{\rho} V\right)^{2}+\frac{1}{4}\left(\partial_{D} V\right)^{2}\right] \geq \frac{9}{7} .
$$

This observation tells us that in the absence of any nongeometric flux $(Q$ and $R$ ), one needs the nonzero Romans mass term along with $A_{\text {loc }}<0$ and $A_{\omega}>0$ in order to avoid the no-go condition [81-83]. Note that the need of $V_{\omega}>0$ is not manifested from the above combination $\left(\partial_{D} V-\rho \partial_{\rho} V\right)$ but can be seen from the previous one as given under

$$
3 \partial_{D} V-\rho \partial_{\rho} V=9 V-2 V_{\omega}+\sum_{p=2,4,6} p V_{f_{p}},
$$

and hence if $V_{\omega}<0$, then the previous NoGo-1 condition would continue to hold leading to $\left(3 \partial_{D} V-\rho \partial_{\rho} V\right)>9 V$ and hence $\epsilon_{V}>27 / 13$. Thus we conclude that merely including the geometric flux does not help for the purpose, and there are other things to engineer to avoid the no-go condition. However, let us recall that merely not finding a no-go condition is not guaranteed for the existence of de Sitter vacua as breaking the known no-go is only a necessary but not a sufficient condition for the de Sitter existence.

\section{B. Evading the no-go conditions with nongeometric fluxes}

On the similar lines of analysis we have discussed and continuing with the focus on the dependence in the $(D, \rho)$ plane only, the generic scalar potential can be clubbed in the following form:

$V=V_{h}+V_{\omega}+V_{q}+V_{r}+V_{f_{0}}+V_{f_{2}}+V_{f_{4}}+V_{f_{6}}+V_{\mathrm{loc}}$,

where we have

$$
\begin{aligned}
V_{h} & =\frac{e^{2 D}}{\rho^{3}} A_{h}, & V_{\omega} & =\frac{e^{2 D}}{\rho} A_{\omega}, \\
V_{q} & =e^{2 D} \rho A_{q}, & V_{r} & =e^{2 D} \rho^{3} A_{r}, \\
V_{\mathrm{loc}} & =e^{3 D} A_{\mathrm{loc}}, & V_{f_{p}} & =\frac{e^{4 D}}{\rho^{(p-3)}} A_{f_{p}} .
\end{aligned}
$$


Here as before $A_{h}, A_{\omega}, A_{q}, A_{r}, A_{\mathrm{loc}}$, and $A_{f_{p}}$ s are flux dependent quantities that do not depend on the volume moduli $\rho$ and the dilaton $D$. Now having full generalities at hand, the following conditions hold on the signs of these coefficients,

$$
\begin{aligned}
& A_{h} \geq 0, \quad A_{r} \geq 0, \quad A_{f_{p}} \geq 0, \quad \forall p=0,2,4,6 ; \\
& \operatorname{sign}\left(A_{\omega}\right), \quad \operatorname{sign}\left(A_{q}\right), \quad \text { and } \operatorname{sign}\left(A_{\text {loc }}\right) \quad \text { not fixed. }
\end{aligned}
$$

Subsequently it is easy to observe that the following relations hold,

$$
\begin{aligned}
& \partial_{D} V_{h}=2 V_{h}, \quad \partial_{D} V_{\omega}=2 V_{\omega}, \quad \partial_{D} V_{q}=2 V_{q}, \quad \partial_{D} V_{r}=2 V_{r}, \\
& \partial_{D} V_{\mathrm{loc}}=3 V_{\mathrm{loc}}, \quad \partial_{D} V_{f_{p}}=4 V_{f_{p}}, \quad \forall p \in\{0,2,4,6\} \text {; } \\
& \partial_{\rho} V_{h}=-\frac{3}{\rho} V_{h}, \quad \partial_{\rho} V_{\omega}=-\frac{1}{\rho} V_{\omega}, \quad \partial_{\rho} V_{q}=\frac{1}{\rho} V_{q}, \quad \partial_{\rho} V_{r}=\frac{3}{\rho} V_{r}, \\
& \partial_{\rho} V_{\mathrm{loc}}=0, \quad \partial_{\rho} V_{f_{p}}=\frac{3-p}{\rho} V_{f_{p}}, \quad \forall p \in\{0,2,4,6\} .
\end{aligned}
$$

For studying the swampland conditions for generic cases having nongeometric fluxes, we take the following combination of the scalar potential derivatives,

$$
\begin{aligned}
\left(\alpha \partial_{D} V-\rho \partial_{\rho} V\right)= & (2 \alpha+3) V_{h}+(2 \alpha+1) V_{\omega}+(2 \alpha-1) V_{q}+(2 \alpha-3) V_{r} \\
& +(4 \alpha-3) V_{f_{0}}+(4 \alpha-1) V_{f_{2}}+(4 \alpha+1) V_{f_{4}}+(4 \alpha+3) V_{f_{6}}+3 \alpha V_{\mathrm{loc}} \\
= & 3 \alpha V+(3-\alpha) V_{h}+(1-\alpha) V_{\omega}-(1+\alpha) V_{q}-(3+\alpha) V_{r}+\sum_{p=0}^{2,4,6}(\alpha-3+p) V_{f_{p}}
\end{aligned}
$$

where in the second equality we have eliminated the $V_{\text {loc }}$ piece as it is (one of) the possible negative piece on the right hand side of the equality. Now, given the uncertainties in the signs of various terms as mentioned in Eq. (4.22) it turns out that, unlike the cases we have analyzed without having any nongeometric flux, now it is not obvious if the rhs in the second line of Eq. (4.24) can be guaranteed to be positive semidefinite for some $\alpha>0$. In particular, one can easily verify how the two de Sitter/inflationary no-go conditions revisited earlier can be evaded in the presence of the nongeometric fluxes; e.g., using $\alpha=3$, we get

$$
\begin{aligned}
\left(3 \partial_{D} V-\rho \partial_{\rho} V\right)= & 9 V-2 V_{\omega}-4 V_{q}-6 V_{r}+2 V_{f_{2}} \\
& +4 V_{f_{4}}+6 V_{f_{6}} .
\end{aligned}
$$

Without knowing the explicit form of the full scalar potential depending on all the moduli, axions, and fluxes, and using schematic dependence on the $D$ and $\rho$ moduli, it has been mentioned in [79] that if $V_{\omega}, V_{q}$, and $V_{r}$ all are negative, then the earlier condition NoGo-1 continues to hold even after including the (non)geometric fluxes. However, from our explicit computations in Appendix B now we have seen that $V_{r} \geq 0$, and therefore the condition NoGo-1 is guaranteed to be evaded after including the nongeometric $R$ flux while keeping the $V_{\omega}$ and $V_{q}$ pieces to zero, otherwise one would need to ensure that $V_{\omega}+2 V_{q}+$ $3 V_{r}>0$ holds in order to break the no-go condition.
Similarly if we consider the second case with $\alpha=1$ corresponding to the condition NoGo-2 we get the following:

$$
\begin{aligned}
\left(\partial_{D} V-\rho \partial_{\rho} V\right)= & 3 V+2 V_{h}-2 V_{q}-4 V_{r}-2 V_{f_{0}} \\
& +2 V_{f_{4}}+4 V_{f_{6}} .
\end{aligned}
$$

Therefore it shows that the condition NoGo-2 would hold as long as $V_{q}+2 V_{r}+V_{f_{0}}<0$ is satisfied. This would be unlikely as $V_{r} \geq 0$ and $V_{f_{0}} \geq 0$ and so $V_{q}$ has to be negative and compensate these two positive contributions. Moreover, this analysis also suggests that, unlike the earlier proposal of [82], now one may not need to demand the vanishing of the Romans mass term $V_{f_{0}}$, and the condition NoGo-2 can be also evaded by appropriately turning on the nongeometric $R$ flux.

As a side remark, it is easy to observe that the two cases discussed in the previous subsections can be directly read off from Eqs. (4.24) and (4.6), leading to the following values of the parameters $\alpha, \beta$ along with a bound on the $\epsilon_{V}$ parameter,

$$
\begin{aligned}
& \text { NoGo1: } \alpha=3, \quad \beta=9 \Rightarrow \epsilon_{V} \geq \frac{27}{13}, \\
& \text { NoGo2: } \alpha=1, \quad \beta=3 \Rightarrow \epsilon_{V} \geq \frac{9}{7} .
\end{aligned}
$$




\section{New de Sitter no-go scenarios for rigid nongeometric compactifications}

In this subsection we will explore the possibility of finding some new de Sitter no-go conditions in the context of type IIA setup with frozen complex structure moduli. This corresponds to having the following period vectors,

$$
\mathcal{X}^{0}=1, \quad \mathcal{F}_{0}=-\frac{i}{2} ; \quad i \int_{X_{3}} \Omega \wedge \bar{\Omega}=1,
$$

which subsequently leads to constant components in the moduli space metric given as follows,

$$
\mathcal{M}^{00}=1=\mathcal{M}_{00} .
$$

It is straightforward to convince that this choice of normalization satisfy all the relations presented in Eq. (B6). Subsequently, the total $F$-term and $D$-term contributions to the scalar potential given in Eqs. (B7)(B8) takes the following simpler form:

$$
\begin{aligned}
& V=\frac{e^{2 D}}{2 \mathcal{V}}\left[\mathbb{M}_{0}^{2}+\mho_{a 0} \mho_{b 0}\left(4 \mathcal{V} \mathcal{G}^{a b}-3 t^{a} t^{b}\right)+\mathbb{Q}^{a}{ }_{0} \mathbb{Q}^{b}{ }_{0}\left(4 \mathcal{V} \mathcal{G}_{a b}-3 \sigma_{a} \sigma_{b}\right)+\mathcal{V}^{2} \mathbb{R}_{0}^{2}+6 \mathbb{M}_{0} \mathbb{Q}^{a}{ }_{0} \sigma_{a}+6 \mathcal{V} \mho_{a 0} \mathbb{R}_{0} t^{a}\right] \\
& +\frac{e^{4 D}}{2 \mathcal{V}}\left[\left(\mathbb{G}^{0}\right)^{2}+\mathbb{G}_{a} \mathcal{V} \mathcal{G}^{a b} \mathbb{G}_{b}+\mathbb{G}^{a} \mathcal{V} \mathcal{G}_{a b} \mathbb{G}^{b}+\mathcal{V}^{2} \mathbb{G}_{0}^{2}\right]-2 e^{3 D}\left(\mathbb{U}_{0} \mathbb{G}_{0}-\mho_{a 0} \mathbb{G}^{a}+\mathbb{Q}^{a} \mathbb{G}_{a}-\mathbb{R}_{0} \mathbb{G}^{0}\right), \\
& +\frac{e^{2 D}}{2 \mathcal{V}}\left[\hat{\mho}_{\alpha}{ }^{0} \mathcal{V}\left(\hat{\kappa}_{a \alpha \beta} t^{a}\right)^{-1} \hat{\mho}_{\beta}{ }^{0}+\mathcal{V} \hat{\mathbb{Q}}^{\alpha 0}\left(\hat{\kappa}_{a \alpha \beta} t^{a}\right) \hat{\mathbb{Q}}^{\beta 0}\right]
\end{aligned}
$$

where the "generalized" flux orbits contain the NS-NS and RR axionic moduli, namely the $b^{a}$ and $\xi^{K}$ as seen from Eq. (B4). Let us emphasize here that the above formulation for the scalar potential given in the Eq. (4.29) is generic for the type IIA setups with frozen complex structure moduli, and once a complete set of Bianchi identities is known, one can utilize them for studying the possibilities about having or not having the de Sitter solutions in generic scenario of the rigid type IIA flux compactifications.

Rather than studying it in full generality, we investigate the possibilities for our toroidal setup with $\mathbb{T}^{6} /\left(\mathbb{Z}_{3} \times \mathbb{Z}_{3}\right)$ orientifold, where, e.g., the full set of Bianchi identities are explicitly known to us, and then one may extrapolate our results and speculate for beyond toroidal situations. For our current type IIA setup with $\mathbb{T}^{6} /\left(\mathbb{Z}_{3} \times \mathbb{Z}_{3}\right)$ orientifold compactification, we have $h_{+}^{1,1}=0$ and hence no fluxes with indices $\alpha \in h_{+}^{1,1}$ are allowed to be present, and subsequently there are no $D$ terms generated, $V_{D}=0$. Further, for our toroidal case, $h_{-}^{1,1}=3$ and so $a \in\{1,2,3\}$. In addition, we have the following relations arising from the only nonvanishing triple intersection number being $\kappa_{123}=1$,

$$
\begin{aligned}
\mathcal{V} & =t^{1} t^{2} t^{3}, \quad \sigma_{1}=\frac{1}{2} \kappa_{1}=t^{2} t^{3}, \quad \sigma_{2}=\frac{1}{2} \kappa_{2}=t^{1} t^{3}, \quad \sigma_{3}=\frac{1}{2} \kappa_{3}=t^{1} t^{2}, \\
\kappa_{a b} & =\left(\begin{array}{ccc}
0 & t^{3} & t^{2} \\
t^{3} & 0 & t^{1} \\
t^{2} & t^{1} & 0
\end{array}\right), \quad-4 \mathcal{V} \kappa^{a b}=\left(\begin{array}{ccc}
2\left(t^{1}\right)^{2} & -2 t^{1} t^{2} & -2 t^{1} t^{3} \\
-2 t^{1} t^{2} & 2\left(t^{2}\right)^{2} & -2 t^{2} t^{3} \\
-2 t^{1} t^{3} & -2 t^{2} t^{3} & 2\left(t^{3}\right)^{2}
\end{array}\right), \\
K^{a \bar{b}} & =\left(\begin{array}{ccc}
4\left(t^{1}\right)^{2} & 0 & 0 \\
0 & 4\left(t^{2}\right)^{2} & 0 \\
0 & 0 & 4\left(t^{3}\right)^{2}
\end{array}\right)=4 \mathcal{V} \mathcal{G}^{a b}, \quad K_{a \bar{b}}=\left(\begin{array}{ccc}
\frac{1}{4\left(t^{1}\right)^{2}} & 0 & 0 \\
0 & \frac{1}{4\left(t^{2}\right)^{2}} & 0 \\
0 & 0 & \frac{1}{4\left(t^{3}\right)^{2}}
\end{array}\right)=\frac{1}{4 \mathcal{V}} \mathcal{G}_{a b} .
\end{aligned}
$$

In the absence of any $D$-term contribution in this construction, the total scalar potential arising from the generic fluxes can be given as follows:

$$
\begin{aligned}
V= & \frac{e^{2 D}}{2 \mathcal{V}}\left[\mathbb{\boxplus}_{0}^{2}+\mho_{a 0} \mho_{b 0}\left(4 \mathcal{V} \mathcal{G}^{a b}-3 t^{a} t^{b}\right)+\mathbb{Q}^{a}{ }_{0} \mathbb{Q}^{b}{ }_{0}\left(4 \mathcal{V} \mathcal{G}_{a b}-3 \sigma_{a} \sigma_{b}\right)+\mathcal{V}^{2} \mathbb{R}_{0}^{2}+6 \mathbb{U}_{0} \mathbb{Q}^{a}{ }_{0} \sigma_{a}+6 \mathcal{V} \mho_{a 0} \mathbb{R}_{0} t^{a}\right] \\
& +\frac{e^{4 D}}{2 \mathcal{V}}\left[\left(\mathbb{G}^{0}\right)^{2}+\mathbb{G}_{a} \mathcal{V} \mathcal{G}^{a b} \mathbb{G}_{b}+\mathbb{G}^{a} \mathcal{V} \mathcal{G}_{a b} \mathbb{G}^{b}+\mathcal{V}^{2} \mathbb{G}_{0}^{2}\right]-2 e^{3 D}\left(\mathbb{H}_{0} \mathbb{G}_{0}-\mho_{a 0} \mathbb{G}^{a}+\mathbb{Q}^{a}{ }_{0} \mathbb{G}_{a}-\mathbb{R}_{0} \mathbb{G}^{0}\right) .
\end{aligned}
$$


Thanks to our generalized flux orbits and the generic formulation of the scalar potential using which we have read off the scalar potential in the above compact form as given in Eq. (4.31). It is remarkable that the full scalar potential written in the above four lines has 391 terms, and to have a cross-check, the interested readers can compute the scalar potential in two ways; one from Eq. (4.31) via using moduli space metric given in Eq. (4.30) along with the generalized flux orbits in Eq. (B4) while the other one via using the following Kähler and superpotentials,

$$
\begin{aligned}
K \equiv & 4 D-\ln (8 \mathcal{V})=-4 \ln \left[\left(-i\left(N^{0}-\bar{N}^{0}\right) / 2\right]-\sum_{a=1,2,3} \ln \left[\left(-i\left(T^{a}-\bar{T}^{a}\right)\right],\right.\right. \\
W= & e_{0}+b^{1} e_{1}+b^{2} e_{2}+b^{3} e_{3}+b^{1} b^{2} m^{3}+b^{2} b^{3} m^{1}+b^{3} b^{1} m^{2}+b^{1} b^{2} b^{3} m_{0} \\
& +2 N^{0}\left(H_{0}+b^{1} \omega_{10}+b^{2} \omega_{20}+b^{3} \omega_{30}+b^{1} b^{2} Q^{3}{ }_{0}+b^{2} b^{3} Q^{1}{ }_{0}+b^{3} b^{1} Q^{2}{ }_{0}+b^{1} b^{2} b^{3} R_{0}\right),
\end{aligned}
$$

where the chiral variables are given as $T^{a}=b^{a}+i t^{a}$ and $N^{0}=\frac{\xi^{0}}{2}+i e^{-D}$. Using the particular details (e.g., the moduli space metrics etc.) about this model we can be more specific about the scalar potential terms; for example the generalized $\mathrm{RR}$ piece of the scalar potential takes the following form:

$$
\begin{aligned}
\sum_{p=0,2,4,6} V_{f_{p}} & \equiv \frac{e^{4 D}}{2 \mathcal{V}}\left[\left(\mathbb{G}^{0}\right)^{2}+\mathbb{G}_{a} \mathcal{V} \mathcal{G}^{a b} \mathbb{G}_{b}+\mathbb{G}^{a} \mathcal{V} \mathcal{G}_{a b} \mathbb{G}^{b}+\mathcal{V}^{2} \mathbb{G}_{0}^{2}\right] \\
& =\frac{e^{4 D}}{2 \mathcal{V}}\left[\left(\mathbb{G}^{0}\right)^{2}+\sum_{a=1}^{3}\left(\mathbb{G}_{a}\right)^{2}\left(t^{a}\right)^{2}+\sum_{a=1}^{3}\left(\mathbb{G}^{a}\right)^{2}\left(\sigma_{a}\right)^{2}+\mathcal{V}^{2} \mathbb{G}_{0}^{2}\right] \geq 0 .
\end{aligned}
$$

Let us mention that the Bianchi identities satisfied by the usual fluxes continue to hold after promoting the various flux components to their respective generalized flux orbits as defined in Eq. (B4). Subsequently, the "generalized" flux constraints for the current type IIA setup can be given as follows:

$$
\begin{aligned}
\mho_{10} \mho_{20} & =\mathbb{H}_{0} \mathbb{Q}^{3}, \quad \mho_{20} \mho_{30}=\mathbb{H}_{0} \mathbb{Q}^{1}, \quad & \mho_{30} \mho_{10}=\mathbb{H}_{0} \mathbb{Q}^{2}{ }_{0}, \\
\mathbb{Q}^{1}{ }_{0} \mathbb{Q}^{2}{ }_{0} & =\mathbb{R}_{0} \mho_{30}, \quad \mathbb{Q}^{2}{ }_{0} \mathbb{Q}^{3}{ }_{0}=\mathbb{R}_{0} \mho_{10}, & \mathbb{Q}^{3}{ }_{0} \mathbb{Q}^{1}{ }_{0}=\mathbb{R}_{0} \mho_{20}, \\
\mathbb{H}_{0} \mathbb{R}_{0} & =\mho_{10} \mathbb{Q}^{1}{ }_{0}=\mho_{20} \mathbb{Q}^{2}{ }_{0}=\mho_{30} \mathbb{Q}_{0}^{3} . &
\end{aligned}
$$

This helps us in significantly nullifying the various scalar potential terms. For example, let us consider all the terms of the $(\widetilde{\mho}+\mathbb{Q} \mathbb{Q})$ type, which we denote as $V_{\omega}$,

$$
V_{\omega} \equiv \frac{e^{2 D}}{2 \mathcal{V}}\left[\mho_{a 0} \mho_{b 0}\left(4 \mathcal{V} \mathcal{G}^{a b}-3 t^{a} t^{b}\right)+6 \mathbb{M}_{0} \mathbb{Q}^{a}{ }_{0} \sigma_{a}\right]=\frac{e^{2 D}}{2}\left[\frac{t^{1}\left(\mho_{10}\right)^{2}}{t^{2} t^{3}}+\frac{t^{2}\left(\mho_{20}\right)^{2}}{t^{1} t^{3}}+\frac{t^{3}\left(\mho_{30}\right)^{2}}{t^{1} t^{2}}\right] \geq 0 .
$$

This not only shows that the $\mathbb{A Q}$ terms are canceled by the $\widetilde{\delta}$ terms but also fixes the sign of $V_{\omega}$ to be positive semidefinite. Similarly for the scalar potential terms of $(\widetilde{R}+\mathbb{Q Q})$ type clubbed in $V_{q}$, we get the following simplifications after nullifying many pieces out of the generalized Bianchi identities,

$$
V_{q} \equiv \frac{e^{2 D}}{2 \mathcal{V}}\left[\mathbb{Q}^{a}{ }_{0} \mathbb{Q}^{b}{ }_{0}\left(4 \mathcal{V} \mathcal{G}_{a b}-3 \sigma_{a} \sigma_{b}\right)+6 \mathcal{V} \mho_{a 0} \mathbb{R}_{0} t^{a}\right]=\frac{e^{2 D}}{2}\left[\frac{t^{2} t^{3}}{t^{1}}\left(\mathbb{Q}^{1}{ }_{0}\right)^{2}+\frac{t^{1} t^{3}}{t^{2}}\left(\mathbb{Q}^{2}{ }_{0}\right)^{2}+\frac{t^{1} t^{2}}{t^{3}}\left(\mathbb{Q}^{3}{ }_{0}\right)^{2}\right] \geq 0 .
$$

Now let us define a new set of moduli $\left\{\rho^{1}, \rho^{2}, \rho^{3}\right\}$ out of the two-cycle moduli $\left\{t^{1}, t^{2}, t^{3}\right\}$ in the following manner:

$$
\frac{t^{2} t^{3}}{t^{1}}=\rho^{1}, \quad \frac{t^{1} t^{3}}{t^{2}}=\rho^{2}, \quad \frac{t^{1} t^{2}}{t^{3}}=\rho^{3} \Rightarrow \mathcal{V}=t^{1} t^{2} t^{3}=\rho^{1} \rho^{2} \rho^{3}
$$

Subsequently we find that our type IIA scalar potential arising from the rigid compactification with (non)geometric fluxes as given in Eq. (4.31) reduces to the following form:

$$
V=V_{h}+V_{\omega}+V_{q}+V_{r}+\sum_{p=0,2,4,6} V_{f_{p}}+V_{\mathrm{loc}}
$$


where all pieces except the $V_{\text {loc }}$ are non-negative with their explicit forms given as follows:

$$
\begin{aligned}
V_{h} & =\frac{e^{2 D}}{2 \rho^{1} \rho^{2} \rho^{3}} \mathbb{G}_{0}^{2} \geq 0, \\
V_{\omega} & =\frac{e^{2 D}}{2}\left[\frac{\left(\mho_{10}\right)^{2}}{\rho^{1}}+\frac{\left(\mho_{20}\right)^{2}}{\rho^{2}}+\frac{\left(\mho_{30}\right)^{2}}{\rho^{3}}\right] \\
& \equiv V_{\omega 1}+V_{\omega 2}+V_{\omega 3} \geq 0, \\
V_{q} & =\frac{e^{2 D}}{2}\left[\left(\mathbb{Q}^{1}{ }^{2}\right)^{2} \rho^{1}+\left(\mathbb{Q}^{2}{ }_{0}\right)^{2} \rho^{2}+\left(\mathbb{Q}^{3}{ }_{0}\right)^{2} \rho^{3}\right] \\
& \equiv V_{q 1}+V_{q 2}+V_{q 3} \geq 0, \\
V_{r} & =\frac{e^{2 D}}{2} \rho^{1} \rho^{2} \rho^{3} \mathbb{R}_{0}^{2} \geq 0, \\
V_{f_{0}} & =\frac{e^{4 D}}{2} \rho^{1} \rho^{2} \rho^{3} \mathbb{G}_{0}^{2} \geq 0, \\
V_{f_{2}} & =\frac{e^{4 D}}{2}\left[\left(\mathbb{G}^{1}\right)^{2} \rho^{1}+\left(\mathbb{G}^{2}\right)^{2} \rho^{2}+\left(\mathbb{G}^{3}\right)^{2} \rho^{3}\right] \\
& \equiv V_{f_{21}}+V_{f_{22}}+V_{f_{23}} \geq 0, \\
V_{f_{4}} & =\frac{e^{4 D}}{2}\left[\frac{\left(\mathbb{G}_{1}\right)^{2}}{\rho^{1}}+\frac{\left(\mathbb{G}_{2}\right)^{2}}{\rho^{2}}+\frac{\left(\mathbb{G}_{3}\right)^{2}}{\rho^{3}}\right] \\
& \equiv V_{f_{41}}+V_{f_{42}}+V_{f_{43}} \geq 0, \\
V_{f_{6}} & =\frac{e^{4 D}}{2 \rho^{1} \rho^{2} \rho^{3}}\left(\mathbb{G}^{0}\right)^{2} \geq 0, \\
V_{\mathrm{loc}} & =-2 e^{3 D}\left(\mathbb{G}_{0} \mathbb{G}_{0}-\sum_{a=1}^{3} \mho_{a 0} \mathbb{G}^{a}+\sum_{a=1}^{3} \mathbb{Q}_{0}^{a} \mathbb{G}_{a}-\mathbb{R}_{0} \mathbb{G}^{0}\right) \\
& <0 .
\end{aligned}
$$

Now let us recall that in order to avoid the de Sitter no-go case we have invoked two conditions: $V_{\omega}+2 V_{q}+3 V_{r}>$ 0 and the other one being $V_{q}+2 V_{r}+V_{f_{0}}>0$. In our current example, we have all the pieces positive except the local term $V_{\text {loc }}$, and therefore if moduli stabilization can be made in a trustworthy regime, there is a possibility of finding a de Sitter minimum. On these lines, now our aim is to investigate if there exist some de Sitter solution, and also the possible new de Sitter no-go conditions with a given particular choice of the fluxes.

After simplifying the scalar potential explicitly, one can convince that the Bianchi identities in Eq. (4.34) written in terms of the generalized flux orbits are equivalent to the following correlations among the various pieces:

$$
\begin{aligned}
& V_{\omega 1} V_{\omega 2}=V_{h} V_{q 3}, \quad V_{\omega 2} V_{\omega 3}=V_{h} V_{q 1}, \quad V_{\omega 1} V_{\omega 3}=V_{h} V_{q 2}, \\
& V_{q 1} V_{q 2}=V_{r} V_{\omega 3}, \quad V_{q 2} V_{q 3}=V_{r} V_{\omega 1}, \quad V_{q 1} V_{q 3}=V_{r} V_{\omega 2} \text {, } \\
& V_{h} V_{r}=V_{\omega 1} V_{q 1}=V_{\omega 2} V_{q 2}=V_{\omega 3} V_{q 3} \text {. }
\end{aligned}
$$

This is quite peculiar simplification for this model. Although we have nullified some terms in the scalar potential via directly using the Bianchi identities, it is still possible that there would be further simplifications once we consider the explicit flux solutions allowed by the Bianchi identities given in Eq. (3.12). We have classified the solutions of these identities into eight types as presented in Eq. (3.13). Moreover, let us also mention that, as we have argued, the Bianchi identities with usual fluxes presented in Eq. (3.12) can be simply promoted to the ones given in Eq. (4.34) using generalized flux orbits. These can be further used in simplifying the potential and for any phenomenological purpose because of the scalar potential being compactly written out in terms of the generalized flux orbits. In addition, given that these generalized flux orbits depend only on fluxes and axions and not on the saxions, we can simply use the solutions of Bianchi identities given in Eq. (3.13) by promoting them to the generalized flux orbits. As axions do not interfere with our saxionic analysis and the approach to invoke or evade de Sitter no-go conditions via looking at some inequalities arising from the extremization of the saxions, it is justified to take the axionic flux orbits as just fluxes satisfying the relations in (4.34) and having the following solutions:

$$
\begin{aligned}
& \text { S1: } \mho_{10}=\mho_{20}=\mho_{30}=0, \quad \mathbb{Q}^{1}{ }_{0}=\mathbb{Q}^{2}{ }_{0}=\mathbb{Q}^{3}{ }_{0}=0, \quad \mathbb{R}_{0}=0 ; \\
& \text { S2: } \mathbb{W}_{0}=0, \quad \mho_{10}=\mho_{20}=\mho_{30}=0, \quad \mathbb{Q}^{1}{ }_{0}=\mathbb{Q}^{2}{ }_{0}=\mathbb{Q}^{3}{ }_{0}=0 ; \\
& \text { S3: } \mathbb{R}_{0}=0, \quad \mathbb{Q}^{1}{ }_{0}=\mathbb{Q}^{2}{ }_{0}=\mathbb{Q}^{3}{ }_{0}=0, \quad \mho_{20}=0=\mho_{30}, \quad \mho_{10} \neq 0 ; \\
& \mathbb{R}_{0}=0, \quad \mathbb{Q}^{1}{ }_{0}=\mathbb{Q}^{2}{ }_{0}=\mathbb{Q}^{3}{ }_{0}=0, \quad \mho_{10}=0=\mho_{30}, \quad \mho_{20} \neq 0 ; \\
& \mathbb{R}_{0}=0, \quad \mathbb{Q}^{1}{ }_{0}=\mathbb{Q}^{2}{ }_{0}=\mathbb{Q}^{3}{ }_{0}=0, \quad \mho_{10}=0=\mho_{20}, \quad \mho_{30} \neq 0 ; \\
& \text { S4: } \mathbb{I}_{0}=0, \quad \mho_{10}=\mho_{20}=\mho_{30}=0, \quad \mathbb{Q}^{2}{ }_{0}=0=\mathbb{Q}^{3}{ }_{0}, \quad \mathbb{Q}^{1}{ }_{0} \neq 0 ; \\
& \mathbb{H}_{0}=0, \quad \mho_{10}=\mho_{20}=\mho_{30}=0, \quad \mathbb{Q}^{1}{ }_{0}=0=\mathbb{Q}^{3}{ }_{0}, \quad \mathbb{Q}^{2}{ }_{0} \neq 0 ; \\
& \mathbb{H}_{0}=0, \quad \mho_{10}=\mho_{20}=\mho_{30}=0, \quad \mathbb{Q}^{1}{ }_{0}=0=\mathbb{Q}^{2}, \quad \mathbb{Q}^{3}{ }_{0} \neq 0 ;
\end{aligned}
$$




\begin{tabular}{|c|c|c|c|c|c|}
\hline 5: $\mathbb{H}_{0}=0$ & $\mho_{20}=0=\mho_{30}$ & $\mathbb{Q}_{0}^{1}=0=\mathbb{Q}_{0}^{2}$ & $\mathbb{R}_{0}=0$ & $\mho_{10} \neq 0$ & $\mathbb{Q}^{3}{ }_{0} \neq c$ \\
\hline $\mathbb{H}_{0}=0$ & $\mho_{10}=0=\mho_{30}$, & $\mathbb{Q}^{1}{ }_{0}=0=\mathbb{Q}_{0}^{2}$ & $\mathbb{R}_{0}=0$ & $\mho_{20} \neq 0$ & $\mathbb{Q}_{0}^{3} \neq$ \\
\hline $\mathbb{H}_{0}=0$, & $\mho_{20}=0=\mho_{30}$, & $\mathbb{Q}_{0}^{1}=0=\mathbb{Q}_{0}^{3}$ & $\mathbb{R}_{0}=0$ & $\mho_{10} \neq 0$ & $\mathbb{Q}^{2}{ }_{0} \neq$ \\
\hline $\mathbb{H}_{0}=0$, & $\mho_{10}=0=\mho_{20}$ & $\mathbb{Q}^{1}{ }_{0}=0=\mathbb{Q}^{3}{ }_{0}$ & $\mathbb{R}_{0}=0$ & $\mho_{30} \neq 0$ & $\mathbb{Q}^{2}{ }_{0} \neq$ \\
\hline $\mathbb{H}_{0}=0$, & $\mho_{10}=0=\mho_{20}$, & $\mathbb{Q}^{2}{ }_{0}=0=\mathbb{Q}_{0}^{3}$ & $\mathbb{R}_{0}=0$ & $\mho_{30} \neq 0$ & $\mathbb{Q}^{1}{ }_{0} \neq$ \\
\hline $\mathrm{H}_{0}=0$ & $\mho_{10}=0=\mho_{30}$, & $\mathbb{Q}_{0}^{2}=0=\mathbb{Q}_{0}^{3}$ & $\mathbb{R}_{0}=0$ & $\mho_{20} \neq 0$ & $\mathbb{Q}_{0}{ }_{0}$ \\
\hline
\end{tabular}

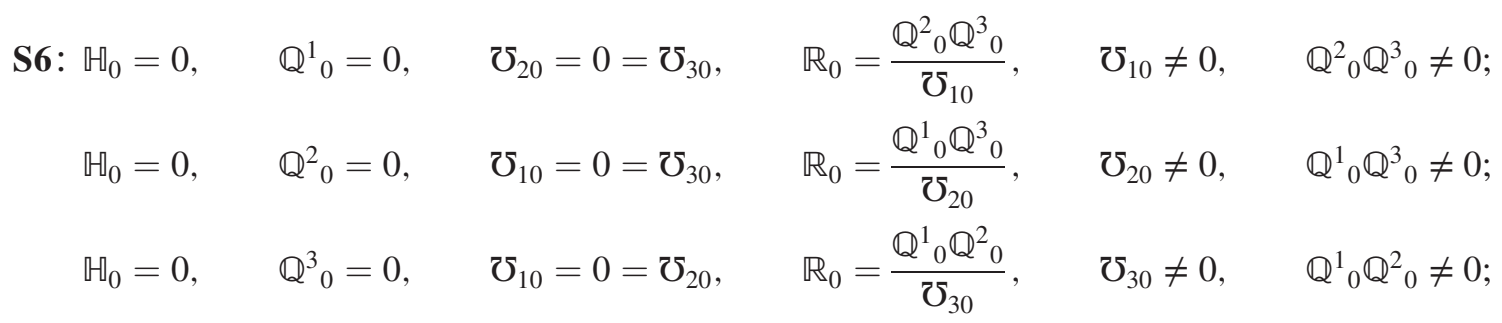

S7: $\mathbb{R}_{0}=0, \quad \mho_{10}=0, \quad \mathbb{Q}^{2}{ }_{0}=0=\mathbb{Q}^{3}{ }_{0}, \quad \mathbb{H}_{0}=\frac{\omega_{20} \mho_{30}}{\mathbb{Q}^{1}{ }_{0}}, \quad \mathbb{Q}^{1}{ }_{0} \neq 0, \quad \mho_{20} \mho_{30} \neq 0 ;$

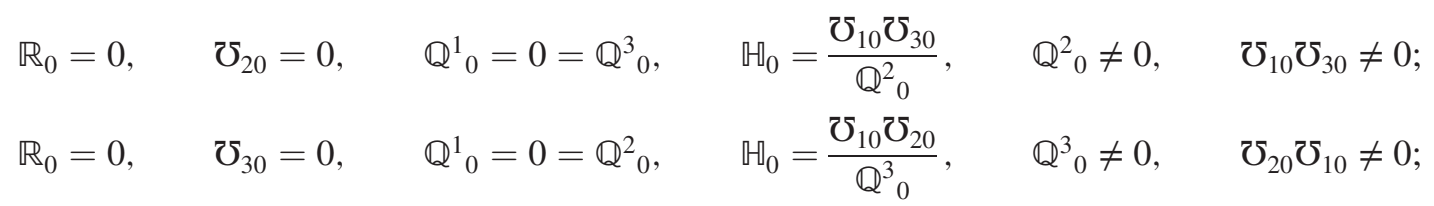

S8: $\left\{\mathbb{Q}_{0}^{1} \neq 0, \quad \mathbb{Q}^{2}{ }_{0} \neq 0, \quad \mathbb{Q}^{3}{ }_{0} \neq 0, \quad \mho_{10}=\frac{\mho_{30} \mathbb{Q}^{3}{ }_{0}}{\mathbb{Q}^{1}{ }_{0}}, \quad \mho_{20}=\frac{\mho_{30} \mathbb{Q}^{3}{ }_{0}}{\mathbb{Q}^{2}{ }_{0}}, \quad \mho_{30} \neq 0\right.$,

$\left.\mathbb{H}_{0}=\frac{\mho_{10} \mho_{20}}{\mathbb{Q}^{3}{ }_{0}}, \quad \mathbb{R}_{0}=\frac{\mathbb{Q}^{1}{ }_{0} \mathbb{Q}^{2}{ }_{0}}{\mho_{30}}\right\}$

We can see that except the class of solutions S8, all the other ones have at least one axionic flux orbit set to zero. Let us make a few comments and observations on the generic solutions of Bianchi identities in Eq. (4.34) when none of the eight NS-NS fluxes, namely $\left\{\mathbb{1}_{0}, \mho_{10}, \mho_{20}, \mho_{30}, \mathbb{Q}_{0}^{1}, \mathbb{Q}^{2}, \mathbb{Q}^{3}{ }_{0}, \mathbb{R}_{0}\right\}$, are zero. In this case, all the nine Bianchi identities in Eq. (4.34) can "effectively" reduce to satisfying the following four constraints:

$$
\begin{aligned}
& \mathbb{Q}^{1}{ }_{0}=\frac{\mho_{20} \mho_{30}}{\mathbb{Z}_{0}}, \quad \mathbb{Q}^{2}{ }_{0}=\frac{\mho_{30} \mho_{10}}{\mathbb{Z}_{0}}, \\
& \mathbb{Q}^{3}{ }_{0}=\frac{\mho_{10} \mho_{20}}{\mathbb{U}_{0}}, \quad \mathbb{R}_{0}=\frac{\mho_{10} \mho_{20} \mho_{30}}{\mathbb{U}_{0}^{2}}
\end{aligned}
$$

Note that this choice of solution is made to show that one could get rid of the purely nongeometric $\left(\mathbb{Q}^{a}{ }_{0}\right.$ and $\left.\mathbb{R}_{0}\right)$ flux orbits in terms of $\mathbb{\sharp}_{0}$ and $\mho_{a 0}$ fluxes. Given that the Bianchi identities satisfied by the set of standard fluxes $\{H, \omega, Q, R\}$ remain to be true after promoting these fluxes to their respective "axionic flux" orbits $\{\mathbb{H}, \mho, \mathbb{Q}, \mathbb{R}\}$ defined in Eq. (B4), we have preferred to work with the latter in which the scalar potential pieces are compactly rewritten as in Eq. (4.39). Having this in mind, the solution in Eq. (4.42) translates into the following correlation among the scalar potential pieces,

$$
\begin{array}{ll}
V_{q 1}=\frac{V_{\omega_{2}} V_{\omega_{3}}}{V_{h}}, & V_{q 2}=\frac{V_{\omega_{3}} V_{\omega_{1}}}{V_{h}}, \\
V_{q 3}=\frac{V_{\omega_{1}} V_{\omega_{2}}}{V_{h}}, & V_{r}=\frac{V_{\omega_{1}} V_{\omega_{2}} V_{\omega_{3}}}{V_{h}^{2}} .
\end{array}
$$

Subsequently the NS-NS pieces can be expressed in the following simplified form:

$$
\begin{aligned}
V_{\mathrm{NS}} & \equiv V_{h}+V_{\omega}+V_{q}+V_{r}, \\
& =V_{h}+V_{\omega_{1}}+V_{\omega_{2}}+V_{\omega_{3}}+V_{q 1}+V_{q 2}+V_{q 3}+V_{r}, \\
& =V_{h}\left(1+\frac{V_{\omega_{1}}}{V_{h}}\right)\left(1+\frac{V_{\omega_{2}}}{V_{h}}\right)\left(1+\frac{V_{\omega_{3}}}{V_{h}}\right) .
\end{aligned}
$$

So we observe that there can be indeed some interesting reshuffling/simplification of the scalar potential pieces induced by the set of Bianchi identities, which could help 
in the search for an analytic study of the possibility of finding the de Sitter (no-go) solutions.

\section{Exploring new de Sitter no-go scenarios}

Now we will separately take each of the eight solutions of the Bianchi identities in Eq. (4.41) to check if there exist a no-go condition for realizing de Sitter vacua. For that purpose, we will simply consider the following three steps:

(1) First, we solve for extremization conditions for all the four saxionic moduli, namely $\left\{\rho^{1}, \rho^{2}, \rho^{3}\right\}$ and $D$ via

$$
\begin{aligned}
\partial_{D} V & =0, & \rho^{1} \partial_{\rho^{1}} V & =0, \\
\rho^{2} \partial_{\rho^{2}} V & =0, & \rho^{3} \partial_{\rho^{3}} V & =0,
\end{aligned}
$$

which leads to identification of some of the scalar potential pieces when evaluated at the extremum.

(2) Second, we evaluate the potential at the extremum using the identification of pieces from step 1 , and we check if that is nonpositive or not. In case it is, we are done, otherwise we further go to step 3.

(3) Third, we evaluate the trace and the determinant of the $4 \times 4$ Hessian at the critical point to check if they are positive or not.

Let us demonstrate this procedure for one of the cases in detail, say with the third solution of S3 to illustrate the steps explicitly and then we will directly tabulated the results for all the solutions. For this case the only nonzero NS-NS flux orbit we have is $\mho_{30} \neq 0$, which leads to

$$
V_{\omega 1}=0=V_{\omega 2}, \quad V_{q}=0, \quad V_{r}=0 .
$$

This can be also understood from the relations in Eq. (4.40) which follows from the Bianchi identities given in Eq. (4.34). Subsequently, the extremization conditions can be translated into the following four constraints,

$$
\begin{aligned}
V_{\mathrm{loc}} & =-\frac{2}{3}\left(4 V_{f_{22}}+4 V_{f_{6}}+4 V_{f_{41}}+4 V_{f_{43}}+3 V_{h}+3 V_{\omega 3}\right), \\
V_{f_{0}} & =-V_{f_{22}}+V_{f_{6}}+V_{f_{42}}+V_{h}, V_{f_{21}}=V_{f_{22}}+V_{f_{41}}-V_{f_{42}}, \\
V_{f_{23}} & =V_{f_{22}}-V_{f_{42}}+V_{f_{43}}+V_{\omega 3} .
\end{aligned}
$$

Using the above conditions in the scalar potential leads to the following form at the extremum:

$$
V_{\mathrm{ext}}=-\frac{2}{3}\left(V_{f_{22}}+V_{f_{6}}+V_{f_{41}}+V_{f_{43}}\right) \leq 0,
$$

which ensures the no-go for the de Sitter vacua. We have applied this technique to conclude that all of the flux solutions except the S8 result in the no-go scenarios for de Sitter realization. Moreover one can also derive the swampland inequalities using the derivatives of the potential, which are given as follows:

$$
\begin{aligned}
\partial_{D} V & =2\left(V_{h}+V_{\omega}+V_{q}+V_{r}\right)+3 V_{\mathrm{loc}}+4 V_{f}, \\
-\rho^{1} \partial_{\rho^{1}} V & =V_{h}+V_{\omega 1}-V_{q 1}-V_{r}-V_{f_{0}}-V_{f_{21}}+V_{f_{41}}+V_{f_{6}}, \\
-\rho^{2} \partial_{\rho^{2}} V & =V_{h}+V_{\omega 2}-V_{q 2}-V_{r}-V_{f_{0}}-V_{f_{22}}+V_{f_{42}}+V_{f_{6}}, \\
-\rho^{3} \partial_{\rho^{3}} V & =V_{h}+V_{\omega 3}-V_{q 3}-V_{r}-V_{f_{0}}-V_{f_{23}}+V_{f_{43}}+V_{f_{6}} .
\end{aligned}
$$

Given that all the pieces in our scalar potential are nonnegative except for the piece $V_{\text {loc }}$, and therefore we can cook many possible scenarios in which de Sitter no-go conditions can be derived for some particular set of flux choices. For example, we can take the following combinations of the scalar potential derivatives that would be useful:

$$
\begin{aligned}
& 3 \partial_{D} V-\sum_{a=1,2,3} \rho^{a} \partial_{\rho^{a}} V \\
& =9 V-2 V_{\omega}-4 V_{q}-6 V_{r}+\sum_{p=0,2,4,6} p V_{f_{p}}, \\
& 3 \partial_{D} V+\sum_{a=1,2,3} \rho^{a} \partial_{\rho^{a}} V \\
& =9 V-2 V_{q}-4 V_{\omega}-6 V_{h}+\sum_{p=0,2,4,6}(6-p) V_{f_{p}} .
\end{aligned}
$$

With some efforts, one leads to the following inequalities resulting into the de Sitter no-go conditions for their respective flux solutions,

$$
\begin{array}{cl}
\text { S1: } 3 \partial_{D} V-\rho^{1} \partial_{\rho^{1}} V-\rho^{2} \partial_{\rho^{2}} V-\rho^{3} \partial_{\rho^{3}} V=9 V+\sum_{p=0,2,4,6} p V_{f_{p}}>9 V, \\
\text { S2: } 3 \partial_{D} V+\rho^{1} \partial_{\rho^{1}} V+\rho^{2} \partial_{\rho^{2}} V+\rho^{3} \partial_{\rho^{3}} V=9 V+\sum_{p=0,2,4,6}(6-p) V_{f_{p}}>9 V, \\
\text { S3: } \partial_{D} V-\rho^{1} \partial_{\rho^{1}} V=3 V+V_{f_{22}}+V_{f_{23}}+V_{f_{4}}+V_{f_{41}}+2 V_{f_{6}}>3 V, \\
\partial_{D} V-\rho^{2} \partial_{\rho^{2}} V=3 V+V_{f_{21}}+V_{f_{23}}+V_{f_{4}}+V_{f_{42}}+2 V_{f_{6}}>3 V, \\
\partial_{D} V-\rho^{3} \partial_{\rho^{3}} V=3 V+V_{f_{21}}+V_{f_{22}}+V_{f_{4}}+V_{f_{43}}+2 V_{f_{6}}>3 V, \\
\text { S4: } \partial_{D} V+\rho^{1} \partial_{\rho^{1}} V=3 V+2 V_{f_{0}}+V_{f_{2}}+V_{f_{21}}+V_{f_{42}}+V_{f_{43}}>3 V, \\
\partial_{D} V+\rho^{2} \partial_{\rho^{2}} V=3 V+2 V_{f_{0}}+V_{f_{2}}+V_{f_{22}}+V_{f_{41}}+V_{f_{43}}>3 V, \\
\partial_{D} V+\rho^{3} \partial_{\rho^{3}} V=3 V+2 V_{f_{0}}+V_{f_{2}}+V_{f_{23}}+V_{f_{41}}+V_{f_{42}}>3 V,
\end{array}
$$




$$
\begin{aligned}
& \text { S5: } \partial_{D} V+\rho^{3} \partial_{\rho^{3}} V-\rho^{1} \partial_{\rho^{1}} V=3 V+V_{f_{0}}+2 V_{f_{22}}+V_{f_{23}}+2 V_{f_{41}}+V_{f_{43}}+V_{f_{6}}>3 V \text {, } \\
& \partial_{D} V+\rho^{3} \partial_{\rho^{3}} V-\rho^{2} \partial_{\rho^{2}} V=3 V+V_{f_{0}}+V_{f_{21}}+2 V_{f_{22}}+V_{f_{41}}+2 V_{f_{43}}+V_{f_{6}}>3 V, \\
& \partial_{D} V+\rho^{2} \partial_{\rho^{2}} V-\rho^{1} \partial_{\rho^{1}} V=3 V+V_{f_{0}}+V_{f_{21}}+2 V_{f_{23}}+V_{f_{41}}+2 V_{f_{42}}+V_{f_{6}}>3 V \text {, } \\
& \partial_{D} V+\rho^{2} \partial_{\rho^{2}} V-\rho^{3} \partial_{\rho^{3}} V=3 V+V_{f_{0}}+V_{f_{22}}+2 V_{f_{23}}+2 V_{f_{41}}+V_{f_{42}}+V_{f_{6}}>3 V \text {, } \\
& \partial_{D} V+\rho^{1} \partial_{\rho^{1}} V-\rho^{3} \partial_{\rho^{3}} V=3 V+V_{f_{0}}+2 V_{f_{21}}+V_{f_{22}}+V_{f_{42}}+2 V_{f_{43}}+V_{f_{6}}>3 V \text {, } \\
& \partial_{D} V+\rho^{1} \partial_{\rho^{1}} V-\rho^{2} \partial_{\rho^{2}} V=3 V+V_{f_{0}}+2 V_{f_{21}}+V_{f_{23}}+2 V_{f_{42}}+V_{f_{43}}+V_{f_{6}}>3 V, \\
& \text { S6: } \partial_{D} V-\rho^{1} \partial_{\rho^{1}} V+\rho^{2} \partial_{\rho^{2}} V+\rho^{3} \partial_{\rho^{3}} V=3 V+2 V_{f_{0}}+2 V_{f_{22}}+2 V_{f_{23}}+2 V_{f_{41}}>3 V \text {, } \\
& \partial_{D} V+\rho^{1} \partial_{\rho^{1}} V-\rho^{2} \partial_{\rho^{2}} V+\rho^{3} \partial_{\rho^{3}} V=3 V+2 V_{f_{0}}+2 V_{f_{21}}+2 V_{f_{23}}+2 V_{f_{42}}>3 V \text {, } \\
& \partial_{D} V+\rho^{1} \partial_{\rho^{1}} V+\rho^{2} \partial_{\rho^{2}} V-\rho^{3} \partial_{\rho^{3}} V=3 V+2 V_{f_{0}}+2 V_{f_{21}}+2 V_{f_{22}}+2 V_{f_{43}}>3 V \text {, } \\
& \text { S7: } \partial_{D} V+\rho^{1} \partial_{\rho^{1}} V-\rho^{2} \partial_{\rho^{2}} V-\rho^{3} \partial_{\rho^{3}} V=3 V+2 V_{f_{21}}+2 V_{f_{42}}+2 V_{f_{43}}+2 V_{f_{6}}>3 V \text {, } \\
& \partial_{D} V-\rho^{1} \partial_{\rho^{1}} V+\rho^{2} \partial_{\rho^{2}} V-\rho^{3} \partial_{\rho^{3}} V=3 V+2 V_{f_{22}}+2 V_{f_{41}}+2 V_{f_{43}}+2 V_{f_{6}}>3 V \text {, } \\
& \partial_{D} V-\rho^{1} \partial_{\rho^{1}} V-\rho^{2} \partial_{\rho^{2}} V+\rho^{3} \partial_{\rho^{3}} V=3 V+2 V_{f_{23}}+2 V_{f_{41}}+2 V_{f_{42}}+2 V_{f_{6}}>3 V \text {. }
\end{aligned}
$$

It is remarkable that, for all the solutions in Eq. (4.41) except the last one, namely the one denoted as S8, we find de Sitter no-go conditions. For the solution S8, we have performed some numerical investigations with random choice of allowed fluxes, but we could not manage to find any de Sitter vacua using integral fluxes. It would be interesting to perform a comprehensive and systematic numerical analysis to investigate the possibility of the existence of a viable de Sitter solution or an extension of the no-go results for the remaining generic case.

\section{CONCLUSIONS}

In this article we have studied the effects of including nongeometric fluxes in the four-dimensional type II models that arise with compactifications using rigid (Calabi Yau) threefolds. In this regard we have considered an explicit construction using the $\mathbb{T}^{6} /\left(\mathbb{Z}_{3} \times \mathbb{Z}_{3}\right)$ toroidal orbifold. First we have presented the so-called missing Bianchi identities in the four-dimensional $\mathcal{N}=2$ theory before orientifolding the setup to be later studied in explicit type IIA and type IIB context. In type IIB orientifold we have observed that the missing Bianchi identities are quite strong and whenever one wants to have at least one nontrivial component for the $\mathrm{H}_{3}$ flux, say for example needed to fix the universal axiodilaton modulus, there remains no scope for turning on any nongeometric $Q$ flux, and hence getting back to the no-scale structure in which the three Kähler moduli remain unstabilized. Based on these observations we also argue that if these could be promoted to the case of rigid $\mathrm{CY}$ threefolds, then several minimal nongeometric setups with rigid CY manifolds, for example (some) models proposed in $[25,26,28,159]$ might be a problem, though one would certainly need to ensure that the conjectured form of the identities in [69], which is further supported by the current analysis, is indeed true for the beyond toroidal cases.

On the type IIA side, we have investigated the solutions of Bianchi identities in some good detail and have found that seven out of eight classes of solutions result in de Sitter no-go scenarios despite having nongeometric fluxes turned on, though a no-go for the most generic case could not be found. However for this generic case also, in our (limited) numerical search we could not manage to find any de Sitter vacua, and it could be conjectured that finding de Sitter vacua with integer fluxes satisfying all the Bianchi identities should not be possible in models of rigid compactifications with (non)geometric fluxes. We plan to get back to addressing this issue in a comprehensive numerical search in the near future.

\section{ACKNOWLEDGMENTS}

We are grateful to Fernando Quevedo for his kind support and encouragements throughout. We would like to thank David Andriot, Erik Plauschinn, Thomas Van Riet, and Timm Wrase for useful discussions and communications. In addition, we are very thankful to the referee for her or his useful comments and suggestions which have helped in improving the manuscript.

\section{APPENDIX A: INGREDIENTS FOR THE $\mathbb{T}^{6} /\left(\mathbb{Z}_{3} \times \mathbb{Z}_{3}\right)$ ORBIFOLD AND ITS OREINTIFOLDS}

In this section we present the relevant pieces of information on the explicit construction of the $\mathbb{T}^{6} /\left(\mathbb{Z}_{3} \times \mathbb{Z}_{3}\right)$ orbifold [71]. Our aim is to first present the computations about (nongeometric) flux components and moduli surviving under the orbifold action regarding the $\mathcal{N}=2$ nongeometric construction, and subsequently we will 
provide those explicit details for the type IIA and type IIB orientifolds as well.

\section{Fluxes and moduli in the $\mathcal{N}=2$ orbifold compactifications}

Let us start by considering the complexified coordinates on the six torus $\mathbb{T}^{6}$ to be defined as follows,

$$
z^{1}=x^{1}+i x^{2}, \quad z^{2}=x^{3}+i x^{4}, \quad z^{3}=x^{5}+i x^{6},
$$

subject to the periodic conditions,

$z^{i} \sim z^{i}+1 \sim z^{i}+\theta, \quad$ where $\theta=e^{i \pi / 3} ; \quad \forall i=1,2,3$.

This six torus has a $\mathbb{Z}_{3}$ symmetry $\Theta_{1}$ acting in the following manner:

$$
\Theta_{1}:\left(z^{1}, z^{2}, z^{3}\right) \rightarrow\left(\theta^{2} z^{1}, \theta^{2} z^{2}, \theta^{2} z^{3}\right) .
$$

This $\Theta_{1}$ action has 27 fixed points and the subsequent orbifold is a singular limit of a Calabi Yau threefold with Euler character $\chi=72$. Moreover, as analyzed in [168,169] the resulting space has an additional $\mathbb{Z}_{3}$ symmetry acting in the following manner,

$\Theta_{2}:\left(z^{1}, z^{2}, z^{3}\right) \rightarrow\left(\theta^{2} z^{1}+\frac{\theta+1}{3}, \theta^{4} z^{2}+\frac{\theta+1}{3}, z^{3}\right)$,

which does not have any fixed point. Further modding out the six torus with the second $\mathbb{Z}_{3}$ action leads to a singular limit of a Calabi Yau threefold with $\chi=24$ having $9 \mathbb{Z}_{3}$ singularities. To be more specific, the $\mathbb{T}^{6} /\left(\mathbb{Z}_{3} \times \mathbb{Z}_{3}\right)$ orbifold compactification results in a construction with frozen complex structure moduli, i.e., having $h^{2,1}=0$ and $h^{1,1}=12$, which corresponds to three standard Kähler moduli and nine blow-up modes. However, in our current study we will focus only on the untwisted sector and hence for us,

$$
h^{1,1}=3, \quad h^{2,1}=0 .
$$

\section{a. Twist invariant forms and moduli}

Now it is easy to construct the twist invariant forms to represent the various moduli and fluxes present in the theory. For that purpose let us first note that

$\Theta_{1}: d z^{i} \rightarrow \theta^{2} d z^{i}, \quad \Theta_{2}: d z^{i} \rightarrow \theta^{2 i} d z^{i} ; \quad \forall i=1,2,3$.

This leads to the following twist invariant two forms $\mu_{A}$,

$$
\begin{aligned}
& \mu_{1}=(\kappa \sqrt{3})^{1 / 3} i d z^{1} \wedge \overline{d z^{1}}=2(\kappa \sqrt{3})^{1 / 3} d x^{1} \wedge d x^{2}, \\
& \mu_{2}=(\kappa \sqrt{3})^{1 / 3} i d z^{2} \wedge \overline{d z^{2}}=2(\kappa \sqrt{3})^{1 / 3} d x^{3} \wedge d x^{4}, \\
& \mu_{3}=(\kappa \sqrt{3})^{1 / 3} i d z^{3} \wedge \overline{d z^{3}}=2(\kappa \sqrt{3})^{1 / 3} d x^{5} \wedge d x^{6},
\end{aligned}
$$

and the four forms $\tilde{\mu}^{A} \mathrm{~s}$, which are dual to the two forms $\left(\mu_{A}\right)$ are defined as follows:

$$
\begin{aligned}
& \tilde{\mu}^{1}=\left(\frac{3}{\kappa}\right)^{1 / 3}\left(i d z^{2} \wedge \overline{d z^{2}}\right)\left(i d z^{3} \wedge \overline{d z^{3}}\right)=\kappa^{-1} \mu_{2} \wedge \mu_{3}, \\
& \tilde{\mu}^{2}=\left(\frac{3}{\kappa}\right)^{1 / 3}\left(i d z^{3} \wedge \overline{d z^{3}}\right)\left(i d z^{1} \wedge \overline{d z^{1}}\right)=\kappa^{-1} \mu_{3} \wedge \mu_{1}, \\
& \tilde{\mu}^{3}=\left(\frac{3}{\kappa}\right)^{1 / 3}\left(i d z^{1} \wedge \overline{d z^{1}}\right)\left(i d z^{2} \wedge \overline{d z^{2}}\right)=\kappa^{-1} \mu_{1} \wedge \mu_{2} .
\end{aligned}
$$

Here the overall normalization has been fixed as follows:

$$
\begin{aligned}
\int_{\mathbb{T}^{6} /\left(\mathbb{Z}_{3} \times \mathbb{Z}_{3}\right)} \mu_{A} \wedge \tilde{\mu}^{B} & =\delta_{A}{ }^{B}, \\
\int_{\mathbb{T}^{6} /\left(\mathbb{Z}_{3} \times \mathbb{Z}_{3}\right)} \mu_{1} \wedge \mu_{2} \wedge \mu_{3} & =\kappa .
\end{aligned}
$$

Further, the twist-invariant three-form $\Omega$ can be defined as follows,

$$
\Omega=3^{1 / 4} i d z^{1} \wedge d z^{2} \wedge d z^{3},
$$

where the normalization in the above unique holomorphic three form $\Omega$ has been fixed via the following constraint,

$$
i \int_{\mathbb{T}^{6} /\left(\mathbb{Z}_{3} \times \mathbb{Z}_{3}\right)} \Omega \wedge \bar{\Omega}=1,
$$

where we have used $i \int_{\mathbb{T}^{6} /\left(\mathbb{Z}_{3} \times \mathbb{Z}_{3}\right)} d z^{i} \wedge \overline{d z^{i}}=\sqrt{3}$. In terms of real cohomology, the holomorphic three form $\Omega$ can be represented as

$$
\Omega=\frac{1}{\sqrt{2}}\left(\mathcal{A}_{0}+i \mathcal{B}^{0}\right), \quad \int_{\mathbb{T}^{6} /\left(\mathbb{Z}_{3} \times \mathbb{Z}_{3}\right)} \mathcal{A}_{0} \wedge \mathcal{B}^{0}=1 .
$$

With a slight difference to the conventions of [71], for our convenience, we want to get rid of normalization factors to write the volume as $\mathcal{V}=t^{1} t^{2} t^{3}$, i.e., the triple intersection number $\kappa_{123} \equiv \kappa=1$. For that let us rescale the complex coordinates by $d z^{i} \rightarrow 3^{-\frac{1}{12}} d z^{i} ; \quad \forall i=\{1,2,3\}$, which would lead to $i \int_{\mathbb{T}^{6} /\left(\mathbb{Z}_{3} \times \mathbb{Z}_{3}\right)} d z^{i} \wedge \overline{d z^{i}}=3^{2 / 3}$, and subsequently the normalizations can be set as follows:

$\Theta_{1}: d z^{i} \rightarrow \theta^{2} d z^{i}, \quad \Theta_{2}: d z^{i} \rightarrow \theta^{2 i} d z^{i} ; \quad \forall i=1,2,3$.

This leads to the following twist invariant two forms $\mu_{A}$, 


$$
\begin{aligned}
& \mu_{1}=d x^{1} \wedge d x^{2}=\frac{i}{2} d z^{1} \wedge \overline{d z^{1}} \\
& \mu_{2}=d x^{3} \wedge d x^{4}=\frac{i}{2} d z^{2} \wedge \overline{d z^{2}} \\
& \mu_{3}=d x^{5} \wedge d x^{6}=\frac{i}{2} d z^{3} \wedge \overline{d z^{3}}
\end{aligned}
$$

and the four forms $\tilde{\mu}^{A} \mathrm{~s}$, which are dual to the two forms $\left(\mu_{A}\right)$, are defined as follows,

$$
\begin{aligned}
& \tilde{\mu}^{1}=\frac{1}{4}\left(i d z^{2} \wedge \overline{d z^{2}}\right)\left(i d z^{3} \wedge \overline{d z^{3}}\right)=\mu_{2} \wedge \mu_{3}, \\
& \tilde{\mu}^{2}=\frac{1}{4}\left(i d z^{3} \wedge \overline{d z^{3}}\right)\left(i d z^{1} \wedge \overline{d z^{1}}\right)=\mu_{3} \wedge \mu_{1}, \\
& \tilde{\mu}^{3}=\frac{1}{4}\left(i d z^{1} \wedge \overline{d z^{1}}\right)\left(i d z^{2} \wedge \overline{d z^{2}}\right)=\mu_{1} \wedge \mu_{2} .
\end{aligned}
$$

Now the overall normalization has been fixed as follows,

$$
\begin{aligned}
\int_{\mathbb{T}^{6} /\left(\mathbb{Z}_{3} \times \mathbb{Z}_{3}\right)} \mu_{A} \wedge \tilde{\mu}^{B} & =\delta_{A}{ }^{B}, \\
\int_{\mathbb{T}^{6} /\left(\mathbb{Z}_{3} \times \mathbb{Z}_{3}\right)} \mu_{1} \wedge \mu_{2} \wedge \mu_{3} & =1 .
\end{aligned}
$$

Further, the twist-invariant three form $\Omega$ can be defined as follows,

$$
\Omega=i d z^{1} \wedge d z^{2} \wedge d z^{3}=\sum_{\Lambda=0}^{3}\left(\alpha_{\Lambda}+i \beta^{\Lambda}\right),
$$

where the real cohomology bases are represented as follows,

$$
\begin{aligned}
& \alpha_{0}=2 \wedge 4 \wedge 6, \quad \alpha_{1}=-2 \wedge 3 \wedge 5, \\
& \alpha_{2}=-1 \wedge 4 \wedge 5, \quad \alpha_{3}=-1 \wedge 3 \wedge 6, \\
& \beta^{0}=1 \wedge 3 \wedge 5, \quad \beta^{1}=-1 \wedge 4 \wedge 6, \\
& \beta^{2}=-2 \wedge 3 \wedge 6, \quad \beta^{3}=-2 \wedge 4 \wedge 5 .
\end{aligned}
$$

Here the shorthand notations $2 \wedge 4 \wedge 6=d x^{2} \wedge d x^{4} \wedge$ $d x^{6}$ etc. have been used, for which the normalization is given as $\int \alpha_{\Lambda} \wedge \beta^{\Delta}=\delta_{\Lambda}{ }^{\Delta}$. Moreover under the orbifold twists we have only two components of the three form to be nonzero as there are following constraints to be imposed,

$$
\alpha_{0}=\alpha_{1}=\alpha_{2}=\alpha_{3}, \quad \beta^{0}=\beta^{1}=\beta^{2}=\beta^{3},
$$

where the normalization in the unique holomorphic three form $\Omega$ has been fixed via

$$
i \int_{\mathbb{T}^{6} /\left(\mathbb{Z}_{3} \times \mathbb{Z}_{3}\right)} \Omega \wedge \bar{\Omega}=1
$$

In terms of real cohomology, the holomorphic three form $\Omega$ can be represented as

$$
\Omega=\frac{1}{\sqrt{2}}\left(\mathcal{A}_{0}+i \mathcal{B}^{0}\right), \quad \int_{\mathbb{T}^{6} /\left(\mathbb{Z}_{3} \times \mathbb{Z}_{3}\right)} \mathcal{A}_{0} \wedge \mathcal{B}^{0}=1 .
$$

\section{b. Twist invariant fluxes}

For our particular $\mathbb{T}^{6} /\left(\mathbb{Z}_{3} \times \mathbb{Z}_{3}\right)$ orbifold construction, we now come to the discussion on the various flux components which survive under the two orbifold twists:

(1) Out of 20 flux components of $H_{i j k}$, there are only 8 components that are allowed to be nonzero and are further constrained by the following six relations,

$$
\begin{aligned}
& H_{235}=H_{145}=H_{136}=-H_{246}, \\
& H_{146}=H_{236}=H_{245}=-H_{135},
\end{aligned}
$$

which lead to only two independent flux components, namely $H_{246}$ and $H_{135}$.

(2) Out of 90 flux components of $\omega_{i j}{ }^{k}$, there are only 24 components that are allowed to be nonzero and are further constrained by the following 18 relations:

$$
\begin{aligned}
& \omega_{23}{ }^{6}=\omega_{14}{ }^{6}=-\omega_{13}{ }^{5}=\omega_{24}{ }^{5}, \\
& \omega_{23}{ }^{5}=\omega_{14}{ }^{5}=\omega_{13}{ }^{6}=-\omega_{24}{ }^{6}, \\
& \omega_{45}{ }^{2}=\omega_{36}{ }^{2}=-\omega_{35}{ }^{1}=\omega_{46}{ }^{1}, \\
& \omega_{45}{ }^{1}=\omega_{36}{ }^{1}=\omega_{35}{ }^{2}=-\omega_{46}{ }^{2}, \\
& \omega_{61}{ }^{4}=\omega_{52}{ }^{4}=-\omega_{51}{ }^{3}=\omega_{62}{ }^{3}, \\
& \omega_{61}{ }^{3}=\omega_{52}{ }^{3}=\omega_{51}{ }^{4}=-\omega_{62}{ }^{4},
\end{aligned}
$$

which lead to the following six "independent" flux components denoted as

$$
\omega_{46}{ }^{1}, \omega_{62}{ }^{3}, \quad \omega_{24}{ }^{5}, \quad \omega_{24}{ }^{6}, \omega_{46}{ }^{2}, \omega_{62}{ }^{4} .
$$

(3) Out of 90 flux components of $Q_{i}{ }^{j k}$, there are only 24 components that are allowed to be nonzero and are further constrained by the following 18 relations:

$$
\begin{aligned}
& Q_{2}{ }^{36}=Q_{1}{ }^{46}=-Q_{1}{ }^{35}=Q_{2}{ }^{45}, \\
& Q_{2}{ }^{35}=Q_{1}{ }^{45}=Q_{1}{ }^{36}=-Q_{2}{ }^{46}, \\
& Q_{4}{ }^{52}=Q_{3}{ }^{62}=-Q_{3}{ }^{51}=Q_{4}{ }^{61}, \\
& Q_{4}{ }^{51}=Q_{3}{ }^{61}=Q_{3}{ }^{52}=-Q_{4}{ }^{62}, \\
& Q_{6}{ }^{14}=Q_{5}{ }^{24}=-Q_{5}{ }^{13}=Q_{6}{ }^{23}, \\
& Q_{6}{ }^{13}=Q_{5}{ }^{23}=Q_{5}{ }^{14}=-Q_{6}{ }^{24},
\end{aligned}
$$


which lead to the following six "independent" flux components denoted as

$$
Q_{4}{ }^{61}, Q_{6}{ }^{23}, Q_{2}{ }^{45}, Q_{2}{ }^{46}, Q_{4}{ }^{62}, Q_{6}{ }^{24} \text {. }
$$

(4) Out of 20 flux components of $R^{i j k}$, there are only 8 components that are allowed to be nonzero and, are further constrained by the following six relations:

$$
\begin{aligned}
& R^{235}=R^{145}=R^{136}=-R^{246} \\
& R^{146}=R^{236}=R^{245}=-R^{135}
\end{aligned}
$$

which leads to only two independent flux components, namely $R^{246}$ and $R^{135}$.

Moreover the flux conversion relations between the standard and cohomology formulation representations are given as follows:

$$
\begin{array}{rlrl}
H_{0} & =H_{135}, & H^{0}=H_{246}, & \\
\omega_{10} & =-\omega_{46}{ }^{2}, & \omega_{20}=-\omega_{62}{ }^{4}, & \omega_{30}=-\omega_{24}{ }^{6}, \\
\omega_{1}{ }^{0} & =-\omega_{46}{ }^{1}, & \omega_{2}{ }^{0}=-\omega_{62}{ }^{3}, & \omega_{3}{ }^{0}=-\omega_{24}{ }^{5}, \\
Q^{1}{ }^{1} & =Q_{2}{ }^{45}, & Q^{2}{ }_{0}=Q_{4}{ }^{61}, & Q^{3}{ }_{0}=Q_{6}{ }^{23}, \\
Q^{10} & =-Q_{2}{ }^{46}, & Q^{20}=-Q_{4}{ }^{62}, & Q^{30}=-Q_{6}{ }^{24}, \\
R_{0} & =R^{246}, & R^{0}=-R^{135} .
\end{array}
$$

\section{Fluxes and moduli in the $\mathcal{N}=1$ orientifold compactifications}

\section{a. Type IIA using a $\mathbb{T}^{6} /\left(\mathbb{Z}_{3} \times \mathbb{Z}_{3}\right)$ orientifold}

The four-dimensional $\mathcal{N}=1$ type IIA model is constructed with the orientifold of a $\mathbb{T}^{6} /\left(\mathbb{Z}_{3} \times \mathbb{Z}_{3}\right)$ orbifold. For that purpose, we further quotient the orbifold by $\mathcal{O}=\Omega_{p}(-1)^{F_{L}} \sigma$, where $\Omega_{p}$ is the worldsheet parity while $(-1)^{F_{L}}$ corresponds to the left moving fermion number, and the antiholomorphic involution $\sigma$ is defined by the following action on the complex coordinates:

$$
\sigma:\left(z^{1}, z^{2}, z^{3}\right) \rightarrow\left(-\bar{z}^{1},-\bar{z}^{2},-\bar{z}^{3}\right) .
$$

Under the orientifold action $\sigma$, the $(1,1)$ cohomology splits into a trivial even sector and a non-trivial odd sector, i.e., we have $h^{1,1}=h_{-}^{1,1}=3$ and $h_{+}^{1,1}=0$. In our conventions $A=\left\{\alpha \in h_{+}^{1,1}, a \in h_{-}^{1,1}\right\}$. Further one has $\sigma: \Omega \rightarrow \bar{\Omega}$, and therefore $\mathcal{A}_{0}$ is even while $\mathcal{B}^{0}$ is odd under the involution. With some computations it turns out that the surviving
NS-NS flux components and their respective cohomology versions are given as follows:

$$
\begin{array}{rlrl}
H_{0} & =H_{135}, & \omega_{10}=-\omega_{46}{ }^{2}, \\
\omega_{20}=-\omega_{62}{ }^{4} & \omega_{30}=-\omega_{24}{ }^{6}, \\
Q^{1}{ }_{0}=Q_{2}{ }^{45}, & Q^{2}{ }_{0}=Q_{4}{ }^{61}, \\
Q^{3}{ }_{0}=Q_{6}{ }^{23}, & R_{0}=R^{246} .
\end{array}
$$

\section{b. Type IIB using a $\mathbb{T}^{6} /\left(\mathbb{Z}_{3} \times \mathbb{Z}_{3}\right)$ orientifold}

The four-dimensional $\mathcal{N}=1$ type IIB model is constructed with the orientifold of a $\mathbb{T}^{6} /\left(\mathbb{Z}_{3} \times \mathbb{Z}_{3}\right)$ orbifold. For that purpose, we further quotient the orbifold by $\mathcal{O}=$ $\Omega_{p}(-1)^{F_{L}} \sigma$, where $\Omega_{p}$ is the worldsheet parity while $(-1)^{F_{L}}$ corresponds to the left moving fermion number, and the holomorphic involution $\sigma$ is defined by the following action:

$$
\sigma:\left(z^{1}, z^{2}, z^{3}\right) \rightarrow\left(-z^{1},-z^{2},-z^{3}\right) .
$$

Under the orientifold action $\sigma$, the $(1,1)$ cohomology splits into a trivial odd sector and a nontrivial even sector, i.e., we have $h^{1,1}=h_{+}^{1,1}=3$ and $h_{-}^{1,1}=0$, which means that there are three Kähler moduli $T_{\alpha}$ and no odd-moduli $G^{a}$ being present in this construction. Recall that in our conventions $A=\left\{\alpha \in h_{+}^{1,1}, a \in h_{-}^{1,1}\right\}$. Further one has $\sigma: \Omega \rightarrow-\Omega$, and therefore $\mathcal{A}_{0}$ as well as $\mathcal{B}^{0}$ both are odd under the involution. Also given that the NS-NS flux $H_{3}$ is odd, and therefore with some computations it turns out that the surviving NS-NS flux components are given as follows:

$$
\begin{aligned}
H_{0} & =H_{135}, & & Q^{1}{ }_{0}=Q_{2}{ }^{45}, \\
Q^{2}{ }_{0} & =Q_{4}{ }^{61}, & & Q^{3}{ }_{0}=Q_{6}{ }^{23}, \\
H^{0} & =H_{246}, & & Q^{10}=-Q_{2}{ }^{46}, \\
Q^{20} & =-Q_{4}{ }^{62}, & & Q^{30}=-Q_{6}{ }^{24} .
\end{aligned}
$$

\section{APPENDIX B: DETAILS ON THE TYPE IIA SCALAR POTENTIAL}

Within the two-derivative approximations, the fourdimensional $\mathcal{N}=1$ type IIA scalar potential receives two types of contributions, one arising from the $F$-term while the other arising from the $D$-term effects, which have been computed for the generic case in [57] and can be expressed in the following collection,

$$
\begin{aligned}
V_{\mathrm{IIA}} \equiv & V_{F}+V_{D}, \\
= & V_{\mathbb{H H U}}+V_{\mho \mho}+V_{\mathbb{Q Q Q}}+V_{\mathbb{R} \mathbb{R}}+V_{\mathbb{H} \mathbb{Q}}+V_{\mathbb{R} \mho} \\
& +V_{\mathbb{G}^{0} \mathbb{G}^{0}}+V_{\mathbb{G}^{a} \mathbb{G}^{a}}+V_{\mathbb{G}_{a} \mathbb{G}_{a}}+V_{\mathbb{G}_{0} \mathbb{G}_{0}} \\
& +V_{D 6 / O 6}+V_{D},
\end{aligned}
$$

where explicit expressions for the various pieces are summarized as follows: 


$$
\begin{aligned}
& V_{\mathbb{H} \boxplus}=\frac{e^{2 D}}{2 \mathcal{V}}\left[\mathbb{H}_{I} \mathcal{M}^{I J} \dddot{M}_{J}\right], \\
& V_{\mho \mho}=\frac{e^{2 D}}{2 \mathcal{V}}\left[\mho_{a I} \mathcal{M}^{I J} \mho_{b J} t^{a} t^{b}+4 \mho_{a I} \mho_{b J} \mathcal{X}^{I} \mathcal{X}^{J}\left(\mathcal{V} \mathcal{G}^{a b}-t^{a} t^{b}\right)\right], \\
& V_{\mathbb{Q Q}}=\frac{e^{2 D}}{2 \mathcal{V}}\left[\mathbb{Q}^{a}{ }_{I} \mathcal{M}^{I J} \mathbb{Q}^{b}{ }_{J} \sigma_{a} \sigma_{b}\right. \\
& \left.+4 \mathbb{Q}^{a}{ }_{I} \mathbb{Q}^{b}{ }_{J} \mathcal{X}^{I} \mathcal{X}^{J}\left(\mathcal{V} \mathcal{G}_{a b}-\sigma_{a} \sigma_{b}\right)\right], \\
& V_{\mathbb{R} \mathbb{R}}=\frac{e^{2 D}}{2 \mathcal{V}}\left[\mathcal{V}^{2} \mathbb{R}_{I} \mathcal{M}^{I J} \mathbb{R}_{J}\right], \\
& V_{\mathbb{H} \mathbb{Q}}=\frac{e^{2 D}}{2 \mathcal{V}} \times(-2)\left[\mathbb{H}_{I} \mathcal{M}^{I J} \mathbb{Q}^{a}{ }_{J} \sigma_{a}-4 \mathbb{U}_{I} \mathcal{X}^{I} \mathcal{X}^{J} \mathbb{Q}^{a}{ }_{J} \sigma_{a}\right], \\
& V_{\mathbb{R} \mho}=\frac{e^{2 D}}{2 \mathcal{V}} \times(-2) \mathcal{V}\left[\mathbb{R}_{I} \mathcal{M}^{I J} \mho_{a J} t^{a}-4 \mathbb{R}_{I} \mathcal{X}^{I} \mathcal{X}^{J} \mho_{a J} t^{a}\right], \\
& V_{\mathbb{G}_{0} \mathbb{G}_{0}}=\frac{e^{4 D}}{2 \mathcal{V}}\left[\mathcal{V}^{2}\left(\mathbb{G}_{0}\right)^{2}\right], \\
& V_{\mathbb{G}^{a} \mathbb{G}^{a}}=\frac{e^{4 D}}{2 \mathcal{V}}\left[\mathcal{V} \mathbb{G}^{a} \mathcal{G}_{a b} \mathbb{G}^{b}\right], \\
& V_{\mathbb{G}_{a} \mathbb{G}_{a}}=\frac{e^{4 D}}{2 \mathcal{V}}\left[\mathcal{V} \mathbb{G}_{a} \mathcal{G}^{a b} \mathbb{G}_{b}\right], \\
& V_{\mathbb{G}^{0} \mathbb{G}^{0}}=\frac{e^{4 D}}{2 \mathcal{V}}\left(\mathbb{G}^{0}\right)^{2},
\end{aligned}
$$

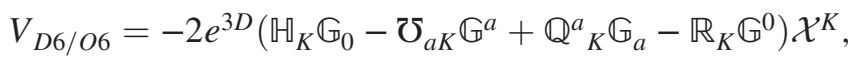

$$
\begin{aligned}
& V_{D}=-2 e^{2 D} \mathcal{F}_{I} \mathcal{F}_{J}\left[\hat{\mho}_{\alpha}{ }^{I} \mathcal{G}^{\alpha \beta} \hat{\boldsymbol{\mho}}_{\beta}{ }^{J}+\hat{\mathbb{Q}}^{\alpha I} \mathcal{G}_{\alpha \beta} \hat{\mathbb{Q}}^{\beta J}\right] \\
& =V_{D}^{(1)}+V_{D}^{(2)} \text {. }
\end{aligned}
$$

Here $D$ denotes the four-dimensional dilaton that is related to the ten-dimensional dilaton $\phi$ via $e^{-D}=e^{-\phi} \sqrt{\mathcal{V}}$ where $\mathcal{V}$ is the volume of the complex threefold. In addition, the various flux orbits involving the axionic moduli $b^{a}$ and $\xi^{K}$ are given as follows:

$$
\begin{aligned}
\mathbb{Q}_{K} & =H_{K}+\omega_{a K} b^{a}+\frac{1}{2} \kappa_{a b c} b^{b} b^{c} Q^{a}{ }_{K}+\frac{1}{6} \kappa_{a b c} b^{a} b^{b} b^{c} R_{K}, \\
\mho_{a K} & =\omega_{a K}+\kappa_{a b c} b^{b} Q^{c}{ }_{K}+\frac{1}{2} \kappa_{a b c} b^{b} b^{c} R_{K}, \\
\mathbb{Q}^{a}{ }_{K} & =Q^{a}{ }_{K}+b^{a} R_{K}, \\
\mathbb{R}_{K} & =R_{K} . \\
\mathbb{G}^{0} & =\mathbb{F}^{0}+\xi^{K} \mathbb{U}_{K}, \\
\mathbb{G}_{a} & =\mathbb{F}_{a}+\xi^{K} \mho_{a K}, \\
\mathbb{G}^{a} & =\mathbb{F}^{a}+\xi^{K} \mathbb{Q}^{a}{ }_{K}, \\
\mathbb{G}_{0} & =\mathbb{F}_{0}+\xi^{K} \mathbb{R}_{K} . \\
\mathbb{F}^{0} & =e^{0}+b^{a} e_{a}+\frac{1}{2} \kappa_{a b c} b^{a} b^{b} m^{c}+\frac{1}{6} \kappa_{a b c} b^{a} b^{b} b^{c} m_{0}, \\
\mathbb{F}_{a} & =e_{a}+\kappa_{a b c} b^{b} m^{c}+\frac{1}{2} \kappa_{a b c} b^{b} b^{c} m_{0}, \\
\mathbb{F}^{a} & =m^{a}+m_{0} b^{a}, \\
\mathbb{F}_{0} & =m_{0} .
\end{aligned}
$$

Moreover, the moduli space metrics follow from [5], and are obtained by orientifolding their parental $\mathcal{N}=2$ metrics given in Eqs. (2.6)-(2.7),

$$
\begin{aligned}
& K_{a \bar{b}}=\frac{\kappa_{a} \kappa_{b}-4 \mathcal{V} \kappa_{a b}}{16 \mathcal{V}^{2}}, \quad K_{a \bar{b}}=\frac{1}{4 \mathcal{V}} \int_{X_{3}} \nu_{a} \wedge * \nu_{b}:=\frac{1}{4 \mathcal{V}} \mathcal{G}_{a b}, \\
& K^{a \bar{b}}=2 t^{a} t^{b}-4 \mathcal{V} \kappa^{a b}, \quad K^{a \bar{b}}=4 \mathcal{V} \int_{X_{3}} \tilde{\nu}^{a} \wedge * \tilde{\mathcal{L}}^{b}:=4 \mathcal{V} \mathcal{G}^{a b}, \\
& K_{\alpha \bar{\beta}}=-\frac{\hat{\kappa}_{\alpha \beta}}{4 \mathcal{V}}, \quad K_{\alpha \bar{\beta}}=\frac{1}{4 \mathcal{V}} \int_{X_{3}} \mu_{\alpha} \wedge * \mu_{\beta}:=\frac{\mathcal{G}_{\alpha \beta}}{4 \mathcal{V}}, \\
& K^{\alpha \bar{\beta}}=-4 \mathcal{V} \hat{\kappa}^{\alpha \beta}, \quad K^{\alpha \bar{\beta}}=4 \mathcal{V} \int_{X_{3}} \tilde{\mu}^{\alpha} \wedge * \tilde{\mu}^{\beta}:=4 \mathcal{V} \mathcal{G}^{\alpha \beta} . \\
& K_{I \bar{J}}=e^{2 D} \mathcal{M}_{I J}, \quad K^{I \bar{J}}=e^{-2 D} \mathcal{M}^{I J},
\end{aligned}
$$

where the shorthand notations such as $\kappa_{a} t^{a}=6 \mathcal{V}$, $\kappa_{a b}=\kappa_{a b c} t^{c}, \kappa_{a}=\kappa_{a b c} t^{b} t^{c}=2 \sigma_{a}$, along with $\kappa^{a b}$ and $\kappa_{a b}$, are used whenever needed. In addition, we have chosen the normalization of the three form such that the following relations are satisfied [82]:

$$
\begin{aligned}
\mathcal{X}^{K} \mathcal{F}_{K}=-\frac{i}{2}, & \mathcal{M}^{I J} \mathcal{F}_{J}=-\frac{i}{2} \mathcal{X}^{I}, \quad \mathcal{M}_{I J} \mathcal{X}^{J}=2 i \mathcal{F}_{I}, \\
\mathcal{M}^{I J} \mathcal{F}_{I} \mathcal{F}_{J} & =-\frac{1}{4}, \quad \mathcal{M}_{I J} \mathcal{X}^{I} \mathcal{X}^{J}=1, \quad \partial_{J} K=-4 e^{D} \mathcal{F}_{J} .
\end{aligned}
$$

Notice that in the absence of (non)geometric fluxes, the collection of scalar potential pieces in Eqs. (B2) reduces into the ones presented, e.g., in Eq. (3.15) of [170] and Eq. (4.26) of [171]. In addition, using the moduli space metric relations in Eq. (B5), it is easy to observe that our scalar potential in Eq. (B2) reduces into the one presented in Eq. (2.34) of [82] when the nongeometric $Q$ and $R$ fluxes are absent but the geometric flux contributions are included.

For the current interest in this work regarding the de Sitter no-go scenarios, we recollect that the scalar potential pieces in Eq. (B1) in some particular manner are given as follows,

$$
\begin{aligned}
V_{\mathrm{IIA}}= & V_{h}+V_{\omega}+V_{q}+V_{r}+V_{f_{0}}+V_{f_{2}} \\
& +V_{f_{4}}+V_{f_{6}}+V_{\mathrm{loc}},
\end{aligned}
$$

where the various pieces can be read off from the Eq. (B2) in the following way:

$$
\begin{aligned}
V_{h} & \equiv V_{\mathbb{H} \mathbb{H}}, \quad V_{\omega} \equiv V_{\mho \mho}+V_{\mathbb{H Q Q}}+V_{D}^{(1)} \\
V_{q} & \equiv V_{\mathbb{Q Q Q}}+V_{\mathbb{R} \mho}+V_{D}^{(2)}, \quad V_{r} \equiv V_{\mathbb{R R}}, \\
V_{f_{0}} & \equiv V_{\mathbb{G}_{0} \mathbb{G}_{0}}, \quad V_{f_{2}} \equiv V_{\mathbb{G}^{a} \mathbb{G}^{a}}, \quad V_{f_{4}} \equiv V_{\mathbb{G}_{a} \mathbb{G}_{a}}, \\
V_{f_{6}} & \equiv V_{\mathbb{G}^{0} \mathbb{G}^{0}}, \quad V_{\mathrm{loc}} \equiv V_{D 6 / O 6} .
\end{aligned}
$$


Here let us mention that we have clubbed the " $H Q$-type" and the " $\omega R$-type" cross terms into what we call $V_{\omega}$ and $V_{q}$ pieces, respectively. As may be obvious from Eq. (B2), this has been done because the " $H Q$ terms" scale similar to " $\omega^{2}$ terms" in two-cycle volume moduli $t^{a}$ and the dilaton $D$, and similarly the " $\omega R$ terms" scale as those of the " $Q^{2}$ terms" in these two moduli. Moreover, the two positive definite $D$-term contributions, which we have denoted as $V_{D}^{(1)}$ and $V_{D}^{(2)}$, are also clubbed along with their respective pieces into $V_{\omega}$ and $V_{q}$, respectively.

Also note the fact that the various pieces in the scalar potential given in Eqs. (B7)-(B8) involve the generalized flux orbits as defined in Eq. (B4), and therefore assuming that $V_{h}$ piece involves only the NS-NS $\mathrm{H}_{3}$-flux components would be misleading as the flux orbit $\mathbb{W}$ can generically have all the NS-NS fluxes, namely the $H, \omega, Q$, and $R$ fluxes. This argument holds true for the other pieces as well. Our observation from the explicit computations of the scalar potential also tells us that the naïve form of the scalar potential as assumed in [85] can be only valid for the cases when the NS-NS axionic moduli $b^{a}$ s are set/stabilized to zero as in that case the generalized flux orbits would reduce into the usual flux components.

We use this scalar potential given in Eqs. (B7)-(B8) for our current analysis in this work. Our generic scalar potential with explicit dependence on all the moduli and the fluxes should enable one to explore more possibilities either for evading or finding new no-go conditions with a different set of flux choices considered in a given scenario.
[1] S. Kachru, R. Kallosh, A. D. Linde, and S. P. Trivedi, De Sitter vacua in string theory, Phys. Rev. D 68, 046005 (2003).

[2] T. R. Taylor and C. Vafa, R R flux on Calabi-Yau and partial supersymmetry breaking, Phys. Lett. B 474, 130 (2000).

[3] R. Blumenhagen, D. Lust, and T. R. Taylor, Moduli stabilization in chiral type IIB orientifold models with fluxes, Nucl. Phys. B663, 319 (2003).

[4] T. W. Grimm and J. Louis, The effective action of type IIA Calabi-Yau orientifolds, Nucl. Phys. B718, 153 (2005).

[5] T. W. Grimm and J. Louis, The effective action of $N=1$ Calabi-Yau orientifolds, Nucl. Phys. B699, 387 (2004).

[6] F. Denef, M. R. Douglas, B. Florea, A. Grassi, and S. Kachru, Fixing all moduli in a simple f-theory compactification, Adv. Theor. Math. Phys. 9, 861 (2005).

[7] M. Grana, Flux compactifications in string theory: A comprehensive review, Phys. Rep. 423, 91 (2006).

[8] V. Balasubramanian, P. Berglund, J. P. Conlon, and F. Quevedo, Systematics of moduli stabilisation in CalabiYau flux compactifications, J. High Energy Phys. 03 (2005) 007.

[9] R. Blumenhagen, B. Kors, D. Lust, and S. Stieberger, Four-dimensional string compactifications with D-branes, orientifolds and fluxes, Phys. Rep. 445, 1 (2007).

[10] M. R. Douglas and S. Kachru, Flux compactification, Rev. Mod. Phys. 79, 733 (2007).

[11] R. Blumenhagen, S. Moster, and E. Plauschinn, Moduli stabilisation versus chirality for MSSM like Type IIB orientifolds, J. High Energy Phys. 01 (2008) 058.

[12] G. Aldazabal, P. G. Camara, A. Font, and L. Ibanez, More dual fluxes and moduli fixing, J. High Energy Phys. 05 (2006) 070.

[13] M. Ihl and T. Wrase, Towards a realistic type IIA $\mathrm{T}^{* * 6 / Z(4)}$ orientifold model with background fluxes. Part 1. Moduli stabilization, J. High Energy Phys. 07 (2006) 027.
[14] M. Ihl, D. Robbins, and T. Wrase, Toroidal orientifolds in IIA with general NS-NS fluxes, J. High Energy Phys. 08 (2007) 043.

[15] A. Font, A. Guarino, and J. M. Moreno, Algebras and nongeometric flux vacua, J. High Energy Phys. 12 (2008) 050.

[16] A. Guarino and G. J. Weatherill, Non-geometric flux vacua, S-duality and algebraic geometry, J. High Energy Phys. 02 (2009) 042.

[17] G. Aldazabal, P. G. Camara, and J. Rosabal, Flux algebra, Bianchi identities and Freed-Witten anomalies in F-theory compactifications, Nucl. Phys. B814, 21 (2009).

[18] B. de Carlos, A. Guarino, and J. M. Moreno, Complete classification of Minkowski vacua in generalised flux models, J. High Energy Phys. 02 (2010) 076.

[19] U. Danielsson and G. Dibitetto, On the distribution of stable de Sitter vacua, J. High Energy Phys. 03 (2013) 018.

[20] J. Blåbäck, U. Danielsson, and G. Dibitetto, Fully stable dS vacua from generalised fluxes, J. High Energy Phys. 08 (2013) 054.

[21] C. Damian and O. Loaiza-Brito, More stable de Sitter vacua from S-dual nongeometric fluxes, Phys. Rev. D 88, 046008 (2013).

[22] C. Damian, L. R. Diaz-Barron, O. Loaiza-Brito, and M. Sabido, Slow-roll inflation in non-geometric flux compactification, J. High Energy Phys. 06 (2013) 109.

[23] F. Hassler, D. Lust, and S. Massai, On inflation and de Sitter in non-geometric string backgrounds, Fortschr. Phys. 65, 1700062 (2017).

[24] R. Blumenhagen and E. Plauschinn, Towards universal axion inflation and reheating in string theory, Phys. Lett. B 736, 482 (2014).

[25] R. Blumenhagen, A. Font, M. Fuchs, D. Herschmann, and E. Plauschinn, Towards axionic Starobinsky-like inflation in string theory, Phys. Lett. B 746, 217 (2015).

[26] R. Blumenhagen, A. Font, M. Fuchs, D. Herschmann, and E. Plauschinn, Large field inflation and string moduli 
stabilization, Proc. Sci., PLANCK2015 (2015) 021 [arXiv: 1510.04059].

[27] T. Li, Z. Li, and D. V. Nanopoulos, Helical phase inflation via non-geometric flux compactifications: From natural to Starobinsky-like inflation, J. High Energy Phys. 10 (2015) 138.

[28] R. Blumenhagen, A. Font, M. Fuchs, D. Herschmann, E. Plauschinn, Y. Sekiguchi, and F. Wolf, A flux-scaling scenario for high-scale moduli stabilization in string theory, Nucl. Phys. B897, 500 (2015).

[29] R. Blumenhagen, C. Damian, A. Font, D. Herschmann, and R. Sun, The flux-scaling scenario: de Sitter uplift and axion inflation, Fortschr. Phys. 64, 536 (2016).

[30] J. Blåbäck, U.H. Danielsson, G. Dibitetto, and S.C. Vargas, Universal dS vacua in STU-models, J. High Energy Phys. 10 (2015) 069.

[31] S. Hellerman, J. McGreevy, and B. Williams, Geometric constructions of nongeometric string theories, J. High Energy Phys. 01 (2004) 024.

[32] A. Dabholkar and C. Hull, Duality twists, orbifolds, and fluxes, J. High Energy Phys. 09 (2003) 054.

[33] C. Hull, A geometry for non-geometric string backgrounds, J. High Energy Phys. 10 (2005) 065.

[34] J.-P. Derendinger, C. Kounnas, P. M. Petropoulos, and F. Zwirner, Superpotentials in IIA compactifications with general fluxes, Nucl. Phys. B715, 211 (2005).

[35] J.-P. Derendinger, C. Kounnas, P. Petropoulos, and F. Zwirner, Fluxes and gaugings: $N=1$ effective superpotentials, Fortschr. Phys. 53, 926 (2005).

[36] J. Shelton, W. Taylor, and B. Wecht, Nongeometric flux compactifications, J. High Energy Phys. 10 (2005) 085 .

[37] B. Wecht, Lectures on nongeometric flux compactifications, Classical Quant. Grav. 24, S773 (2007).

[38] G. Dall'Agata, G. Villadoro, and F. Zwirner, Type-IIA flux compactifications and $N=4$ gauged supergravities, J. High Energy Phys. 08 (2009) 018.

[39] G. Aldazabal, D. Marques, C. Nunez, and J. A. Rosabal, On Type IIB Moduli stabilization and $N=4,8$ supergravities, Nucl. Phys. B849, 80 (2011).

[40] G. Aldazabal, W. Baron, D. Marques, and C. Nunez, The effective action of double field theory, J. High Energy Phys. 11 (2011) 052.

[41] D. Geissbuhler, Double field theory and $N=4$ gauged supergravity, J. High Energy Phys. 11 (2011) 116.

[42] M. Graña and D. Marques, Gauged double field theory, J. High Energy Phys. 04 (2012) 020.

[43] G. Dibitetto, J. Fernandez-Melgarejo, D. Marques, and D. Roest, Duality orbits of non-geometric fluxes, Fortschr. Phys. 60, 1123 (2012).

[44] D. Andriot and A. Betz, $\beta$-supergravity: A ten-dimensional theory with non-geometric fluxes, and its geometric framework, J. High Energy Phys. 12 (2013) 083.

[45] D. Andriot and A. Betz, Supersymmetry with nongeometric fluxes, or a $\beta$-twist in generalized geometry and Dirac operator, J. High Energy Phys. 04 (2015) 006.

[46] C. D. A. Blair and E. Malek, Geometry and fluxes of SL(5) exceptional field theory, J. High Energy Phys. 03 (2015) 144.
[47] D. Andriot, O. Hohm, M. Larfors, D. Lust, and P. Patalong, Non-geometric fluxes in supergravity and double field theory, Fortschr. Phys. 60, 1150 (2012).

[48] D. Geissbuhler, D. Marques, C. Nunez, and V. Penas, Exploring double field theory, J. High Energy Phys. 06 (2013) 101.

[49] R. Blumenhagen, X. Gao, D. Herschmann, and P. Shukla, Dimensional oxidation of non-geometric fluxes in type II orientifolds, J. High Energy Phys. 10 (2013) 201.

[50] G. Villadoro and F. Zwirner, $N=1$ effective potential from dual type-IIA D6/O6 orientifolds with general fluxes, J. High Energy Phys. 06 (2005) 047.

[51] D. Robbins and T. Wrase, D-terms from generalized NS-NS fluxes in type II, J. High Energy Phys. 12 (2007) 058 .

[52] D. M. Lombardo, F. Riccioni, and S. Risoli, $P$ fluxes and exotic branes, J. High Energy Phys. 12 (2016) 114.

[53] D. M. Lombardo, F. Riccioni, and S. Risoli, Nongeometric fluxes \& tadpole conditions for exotic branes, J. High Energy Phys. 10 (2017) 134.

[54] A. Ceresole, R. D'Auria, and S. Ferrara, The symplectic structure of $N=2$ supergravity and its central extension, Nucl. Phys. B, Proc. Suppl. 46, 67 (1996).

[55] R. D'Auria, S. Ferrara, and M. Trigiante, On the supergravity formulation of mirror symmetry in generalized Calabi-Yau manifolds, Nucl. Phys. B780, 28 (2007).

[56] P. Shukla, A symplectic rearrangement of the four dimensional non-geometric scalar potential, J. High Energy Phys. 11 (2015) 162.

[57] X. Gao, P. Shukla, and R. Sun, Symplectic formulation of the type IIA nongeometric scalar potential, Phys. Rev. D 98, 046009 (2018).

[58] P. Shukla, Reading off the nongeometric scalar potentials via the topological data of the compactifying Calabi-Yau manifolds, Phys. Rev. D 94, 086003 (2016).

[59] X. Gao and P. Shukla, Dimensional oxidation and modular completion of non-geometric type IIB action, J. High Energy Phys. 05 (2015) 018.

[60] P. Shukla, On modular completion of generalized flux orbits, J. High Energy Phys. 11 (2015) 075.

[61] P. Shukla, Implementing odd-axions in dimensional oxidation of $4 \mathrm{D}$ non-geometric type IIB scalar potential, Nucl. Phys. B902, 458 (2016).

[62] D. Andriot, O. Hohm, M. Larfors, D. Lust, and P. Patalong, A Geometric Action for Non-Geometric Fluxes, Phys. Rev. Lett. 108, 261602 (2012).

[63] D. Andriot, M. Larfors, D. Lust, and P. Patalong, A tendimensional action for non-geometric fluxes, J. High Energy Phys. 09 (2011) 134.

[64] R. Blumenhagen, A. Font, and E. Plauschinn, Relating double field theory to the scalar potential of $N=2$ gauged supergravity, J. High Energy Phys. 12 (2015) 122.

[65] E. Plauschinn, Non-geometric backgrounds in string theory, Phys. Rep. 798, 1 (2019).

[66] I. Benmachiche and T.W. Grimm, Generalized $N=1$ orientifold compactifications and the Hitchin functionals, Nucl. Phys. B748, 200 (2006).

[67] M. Grana, J. Louis, and D. Waldram, $\mathrm{SU}(3) \times \mathrm{SU}(3)$ compactification and mirror duals of magnetic fluxes, J. High Energy Phys. 04 (2007) 101. 
[68] P. Shukla, Revisiting the two formulations of Bianchi identities and their implications on moduli stabilization, J. High Energy Phys. 08 (2016) 146.

[69] X. Gao, P. Shukla, and R. Sun, On missing Bianchi identities in cohomology formulation, Eur. Phys. J. C 79, 781 (2019).

[70] P. Betzler and E. Plauschinn, Type IIB flux vacua and tadpole cancellation, Fortschr. Phys. 67, 1900065 (2019).

[71] O. DeWolfe, A. Giryavets, S. Kachru, and W. Taylor, Type IIA moduli stabilization, J. High Energy Phys. 07 (2005) 066.

[72] P. G. Camara, A. Font, and L. E. Ibanez, Fluxes, moduli fixing and MSSM-like vacua in a simple IIA orientifold, J. High Energy Phys. 09 (2005) 013.

[73] E. Palti, G. Tasinato, and J. Ward, WEAKLY-coupled IIA flux compactifications, J. High Energy Phys. 06 (2008) 084.

[74] G. Dibitetto, A. Guarino, and D. Roest, Vacua analysis in extended supersymmetry compactifications, Fortschr. Phys. 60, 987 (2012).

[75] J. Blåbäck, U. Danielsson, and G. Dibitetto, Accelerated Universes from type IIA compactifications, J. Cosmol. Astropart. Phys. 03 (2014) 003.

[76] D. Escobar, F. Marchesano, and W. Staessens, Type IIA flux vacua and $\alpha^{\prime}$-corrections, J. High Energy Phys. 06 (2019) 129.

[77] F. Marchesano and J. Quirant, A landscape of AdS flux vacua, J. High Energy Phys. 12 (2019) 110.

[78] J. M. Maldacena and C. Nunez, Supergravity description of field theories on curved manifolds and a no go theorem, Int. J. Mod. Phys. A 16, 822 (2001).

[79] M. P. Hertzberg, S. Kachru, W. Taylor, and M. Tegmark, Inflationary constraints on type IIA string theory, J. High Energy Phys. 12 (2007) 095.

[80] M. P. Hertzberg, M. Tegmark, S. Kachru, J. Shelton, and O. Ozcan, Searching for inflation in simple string theory models: An astrophysical perspective, Phys. Rev. D 76, 103521 (2007).

[81] S. S. Haque, G. Shiu, B. Underwood, and T. Van Riet, Minimal simple de Sitter solutions, Phys. Rev. D 79, 086005 (2009).

[82] R. Flauger, S. Paban, D. Robbins, and T. Wrase, Searching for slow-roll moduli inflation in massive type IIA supergravity with metric fluxes, Phys. Rev. D 79, 086011 (2009).

[83] C. Caviezel, P. Koerber, S. Kors, D. Lust, T. Wrase, and M. Zagermann, On the cosmology of Type IIA compactifications on SU(3)-structure manifolds, J. High Energy Phys. 04 (2009) 010.

[84] L. Covi, M. Gomez-Reino, C. Gross, J. Louis, G. A. Palma, and C. A. Scrucca, de Sitter vacua in no-scale supergravities and Calabi-Yau string models, J. High Energy Phys. 06 (2008) 057.

[85] B. de Carlos, A. Guarino, and J. M. Moreno, Flux moduli stabilisation, Supergravity algebras and no-go theorems, J. High Energy Phys. 01 (2010) 012.

[86] C. Caviezel, T. Wrase, and M. Zagermann, Moduli stabilization and cosmology of type IIB on SU(2)-structure orientifolds, J. High Energy Phys. 04 (2010) 011.
[87] U. H. Danielsson, S. S. Haque, G. Shiu, and T. Van Riet, Towards classical de Sitter solutions in string theory, J. High Energy Phys. 09 (2009) 114.

[88] U. H. Danielsson, P. Koerber, and T. Van Riet, Universal de Sitter solutions at tree-level, J. High Energy Phys. 05 (2010) 090.

[89] T. Wrase and M. Zagermann, On Classical de Sitter Vacua in String Theory, Fortschr. Phys. 58, 906 (2010).

[90] G. Shiu and Y. Sumitomo, Stability constraints on classical de Sitter vacua, J. High Energy Phys. 09 (2011) 052.

[91] J. McOrist and S. Sethi, M-theory and type IIA flux compactifications, J. High Energy Phys. 12 (2012) 122.

[92] K. Dasgupta, R. Gwyn, E. McDonough, M. Mia, and R. Tatar, de Sitter vacua in type IIB string theory: Classical solutions and quantum corrections, J. High Energy Phys. 07 (2014) 054.

[93] F. F. Gautason, M. Schillo, T. Van Riet, and M. Williams, Remarks on scale separation in flux vacua, J. High Energy Phys. 03 (2016) 061.

[94] D. Junghans, Tachyons in classical de Sitter Vacua, J. High Energy Phys. 06 (2016) 132.

[95] D. Andriot and J. Blåbäck, Refining the boundaries of the classical de Sitter landscape, J. High Energy Phys. 03 (2017) 102; Erratum, J. High Energy Phys. 03 (2018) 83.

[96] D. Andriot, On classical de Sitter and Minkowski solutions with intersecting branes, J. High Energy Phys. 03 (2018) 054.

[97] U. H. Danielsson and T. Van Riet, What if string theory has no de Sitter vacua?, Int. J. Mod. Phys. D 27, 1830007 (2018).

[98] H. Ooguri and C. Vafa, On the geometry of the string landscape and the Swampland, Nucl. Phys. B766, 21 (2007).

[99] G. Obied, H. Ooguri, L. Spodyneiko, and C. Vafa, De Sitter space and the Swampland, arXiv:1806.08362.

[100] U. H. Danielsson, S. S. Haque, P. Koerber, G. Shiu, T. Van Riet, and T. Wrase, De Sitter hunting in a classical landscape, Fortschr. Phys. 59, 897 (2011).

[101] X. Chen, G. Shiu, Y. Sumitomo, and S. H. H. Tye, A global view on the search for de Sitter vacua in (type IIA) string theory, J. High Energy Phys. 04 (2012) 026.

[102] U. H. Danielsson, G. Shiu, T. Van Riet, and T. Wrase, A note on obstinate tachyons in classical dS solutions, J. High Energy Phys. 03 (2013) 138.

[103] D. Andriot, On the de Sitter swampland criterion, Phys. Lett. B 785, 570 (2018).

[104] D. Andriot, New constraints on classical de Sitter: Flirting with the swampland, Fortschr. Phys. 67, 1800103 (2019).

[105] S. K. Garg and C. Krishnan, Bounds on slow roll and the de Sitter Swampland, J. High Energy Phys. 11 (2019) 075 .

[106] F. Denef, A. Hebecker, and T. Wrase, de Sitter swampland conjecture and the Higgs potential, Phys. Rev. D 98, 086004 (2018).

[107] J. P. Conlon, The de Sitter swampland conjecture and supersymmetric AdS vacua, Int. J. Mod. Phys. A 33, 1850178 (2018).

[108] C. Roupec and T. Wrase, de Sitter extrema and the Swampland, Fortschr. Phys. 67, 1800082 (2019). 
[109] H. Murayama, M. Yamazaki, and T. T. Yanagida, Do we live in the Swampland?, J. High Energy Phys. 12 (2018) 032.

[110] K. Choi, D. Chway, and C. S. Shin, The dS swampland conjecture with the electroweak symmetry and QCD chiral symmetry breaking, J. High Energy Phys. 11 (2018) 142.

[111] K. Hamaguchi, M. Ibe, and T. Moroi, The Swampland conjecture and the Higgs expectation value, J. High Energy Phys. 12 (2018) 023.

[112] Y. Olguin-Trejo, S. L. Parameswaran, G. Tasinato, and I. Zavala, Runaway quintessence, out of the Swampland, J. Cosmol. Astropart. Phys. 01 (2019) 031.

[113] J. J. Blanco-Pillado, M. A. Urkiola, and J. M. Wachter, Racetrack potentials and the de Sitter Swampland conjectures, J. High Energy Phys. 01 (2019) 187.

[114] H. Ooguri, E. Palti, G. Shiu, and C. Vafa, Distance and de Sitter conjectures on the Swampland, Phys. Lett. B 788, 180 (2019).

[115] J. J. Blanco-Pillado, C. P. Burgess, J. M. Cline, C. Escoda, M. Gomez-Reino, R. Kallosh, A. D. Linde, and F. Quevedo, Inflating in a better racetrack, J. High Energy Phys. 09 (2006) 002.

[116] W. H. Kinney, S. Vagnozzi, and L. Visinelli, The zoo plot meets the swampland: Mutual (in)consistency of singlefield inflation, string conjectures, and cosmological data, Classical Quant. Grav. 36, 117001 (2019).

[117] A. Achúcarro and G. A. Palma, The string Swampland constraints require multi-field inflation, J. Cosmol. Astropart. Phys. 02 (2019) 041.

[118] A. Kehagias and A. Riotto, A note on inflation and the Swampland, Fortschr. Phys. 66, 1800052 (2018).

[119] W. H. Kinney, Eternal Inflation and the Refined Swampland Conjecture, Phys. Rev. Lett. 122, 081302 (2019).

[120] C.-M. Lin, K.-W. Ng, and K. Cheung, Chaotic inflation on the brane and the Swampland Criteria, Phys. Rev. D 100, 023545 (2019).

[121] C. Han, S. Pi, and M. Sasaki, Quintessence saves Higgs instability, Phys. Lett. B 791, 314 (2019).

[122] M. Raveri, W. Hu, and S. Sethi, Swampland conjectures and late-time cosmology, Phys. Rev. D 99, 083518 (2019).

[123] U. Danielsson, The quantum swampland, J. High Energy Phys. 04 (2019) 095.

[124] K. Dasgupta, M. Emelin, E. McDonough, and R. Tatar, Quantum corrections and the de Sitter Swampland conjecture, J. High Energy Phys. 01 (2019) 145.

[125] S. Andriolo, G. Shiu, H. Triendl, T. Van Riet, G. Venken, and G. Zoccarato, Compact G2 holonomy spaces from SU(3) structures, J. High Energy Phys. 03 (2019) 059.

[126] K. Dasgupta, M. Emelin, M. M. Faruk, and R. Tatar, de Sitter vacua in the string landscape, arXiv:1908.05288.

[127] D. Andriot, Open problems on classical de Sitter solutions, Fortschr. Phys. 67, 1900026 (2019).

[128] E. Palti, The Swampland: Introduction and review, Fortschr. Phys. 67, 1900037 (2019).

[129] R. Blumenhagen, I. Valenzuela, and F. Wolf, The Swampland conjecture and F-term axion monodromy inflation, J. High Energy Phys. 07 (2017) 145.

[130] R. Blumenhagen, D. Klaewer, L. Schlechter, and F. Wolf, The refined Swampland distance conjecture in Calabi-Yau Moduli spaces, J. High Energy Phys. 06 (2018) 052.
[131] R. Blumenhagen, Large field inflation/quintessence and the refined Swampland distance conjecture, Proc. Sci., CORFU2017 (2018) 175 [arXiv:1804.10504].

[132] E. Palti, The weak gravity conjecture and scalar fields, J. High Energy Phys. 08 (2017) 034.

[133] J. P. Conlon and S. Krippendorf, Axion decay constants away from the lamppost, J. High Energy Phys. 04 (2016) 085 .

[134] A. Hebecker, P. Henkenjohann, and L. T. Witkowski, Flat monodromies and a moduli space size conjecture, J. High Energy Phys. 12 (2017) 033.

[135] D. Klaewer and E. Palti, Super-Planckian Spatial field variations and quantum gravity, J. High Energy Phys. 01 (2017) 088.

[136] F. Baume and E. Palti, Backreacted axion field ranges in string theory, J. High Energy Phys. 08 (2016) 043.

[137] A. Landete and G. Shiu, Mass hierarchies and dynamical field range, Phys. Rev. D 98, 066012 (2018).

[138] M. Cicoli, D. Ciupke, C. Mayrhofer, and P. Shukla, A geometrical upper bound on the inflaton range, J. High Energy Phys. 05 (2018) 001.

[139] A. Font, A. Herráez, and L. E. Ibáñez, The Swampland distance conjecture and towers of tensionless branes, J. High Energy Phys. 08 (2019) 044.

[140] T. W. Grimm, C. Li, and E. Palti, Infinite distance networks in field space and charge orbits, J. High Energy Phys. 03 (2019) 016.

[141] A. Hebecker, D. Junghans, and A. Schachner, Large field ranges from aligned and misaligned winding, J. High Energy Phys. 03 (2019) 192.

[142] A. Banlaki, A. Chowdhury, C. Roupec, and T. Wrase, Scaling limits of dS vacua and the swampland, J. High Energy Phys. 03 (2019) 065.

[143] D. Junghans, Weakly Coupled de Sitter vacua with fluxes and the Swampland, J. High Energy Phys. 03 (2019) 150 .

[144] C. P. Burgess, R. Kallosh, and F. Quevedo, de Sitter string vacua from supersymmetric D terms, J. High Energy Phys. 10 (2003) 056.

[145] A. Achucarro, B. de Carlos, J. A. Casas, and L. Doplicher, de Sitter vacua from uplifting D-terms in effective supergravities from realistic strings, J. High Energy Phys. 06 (2006) 014.

[146] A. Westphal, de Sitter string vacua from Kahler uplifting, J. High Energy Phys. 03 (2007) 102.

[147] E. Silverstein, Simple de Sitter solutions, Phys. Rev. D 77, 106006 (2008).

[148] M. Rummel and A. Westphal, A sufficient condition for de Sitter vacua in type IIB string theory, J. High Energy Phys. 01 (2012) 020.

[149] M. Cicoli, A. Maharana, F. Quevedo, and C. P. Burgess, de Sitter string vacua from dilaton-dependent nonperturbative effects, J. High Energy Phys. 06 (2012) 011.

[150] J. Louis, M. Rummel, R. Valandro, and A. Westphal, Building an explicit de Sitter, J. High Energy Phys. 10 (2012) 163.

[151] M. Cicoli, D. Klevers, S. Krippendorf, C. Mayrhofer, F. Quevedo, and R. Valandro, Explicit de Sitter flux vacua for global string models with chiral matter, J. High Energy Phys. 05 (2014) 001. 
[152] M. Cicoli, F. Quevedo, and R. Valandro, de Sitter from T-branes, J. High Energy Phys. 03 (2016) 141.

[153] M. Cicoli, I. Garcìa-Etxebarria, C. Mayrhofer, F. Quevedo, P. Shukla, and R. Valandro, Global orientifolded quivers with inflation, J. High Energy Phys. 11 (2017) 134.

[154] Y. Akrami, R. Kallosh, A. Linde, and V. Vardanyan, The landscape, the Swampland and the era of precision cosmology, Fortschr. Phys. 67, 1800075 (2019).

[155] I. Antoniadis, Y. Chen, and G. K. Leontaris, Perturbative moduli stabilisation in type IIB/F-theory framework, Eur. Phys. J. C 78, 766 (2018).

[156] J. J. Heckman, C. Lawrie, L. Lin, J. Sakstein, and G. Zoccarato, Pixelated dark energy, Fortschr. Phys. 67, 1900071 (2019).

[157] J. J. Heckman, C. Lawrie, L. Lin, and G. Zoccarato, F-theory and dark energy, Fortschr. Phys. 67, 1900057 (2019).

[158] M. Cicoli, S. De Alwis, A. Maharana, F. Muia, and F. Quevedo, De Sitter vs quintessence in string theory, Fortschr. Phys. 67, 1800079 (2019).

[159] C. Damian and O. Loaiza-Brito, Two-field axion inflation and the Swampland constraint in the flux-scaling scenario, Fortschr. Phys. 67, 1800072 (2019).

[160] P. Shukla, preceding paper, A dictionary for the type II non-geometric flux compactifications, Phys. Rev. D 103, 086009 (2021).

[161] P. Shukla, T -dualizing de Sitter no-go scenarios, Phys. Rev. D 102, 026014 (2020).
[162] S. Hosono, A. Klemm, and S. Theisen, Lectures on mirror symmetry, Lect. Notes Phys. 436, 235 (1994).

[163] K. Hori and C. Vafa, Mirror symmetry, arXiv:hep-th/ 0002222.

[164] S. Sethi, Supermanifolds, rigid manifolds and mirror symmetry, Nucl. Phys. B430, 31 (1994); AMS/IP Stud. Adv. Math. 1, 793 (1996).

[165] J. Shelton, W. Taylor, and B. Wecht, Generalized flux vacua, J. High Energy Phys. 02 (2007) 095.

[166] G. Aldazabal, D. Marques, and C. Nunez, Double field theory: A pedagogical review, Classical Quant. Grav. 30, 163001 (2013).

[167] D. Andriot and A. Betz, NS-branes, source corrected Bianchi identities, and more on backgrounds with non-geometric fluxes, J. High Energy Phys. 07 (2014) 059 .

[168] L. J. Dixon, J. A. Harvey, C. Vafa, and E. Witten, Strings on orbifolds, Nucl. Phys. B261, 678 (1985).

[169] A. Strominger, Topology of superstring compactification, in Workshop on Unified String Theories Santa Barbara, California, 1985 (1985).

[170] F. Carta, F. Marchesano, W. Staessens, and G. Zoccarato, Open string multi-branched and Kähler potentials, J. High Energy Phys. 09 (2016) 062.

[171] F. Farakos, S. Lanza, L. Martucci, and D. Sorokin, Threeforms in supergravity and flux compactifications, Eur. Phys. J. C 77, 602 (2017). 